\title{
Von der Ekphrasis zur wissenschaftlichen Bildbeschreibung Vasari, Agucchi, Félibien, Burckhardt ${ }^{\star}$
}

\begin{abstract}
Die Frage nach der Beschreibung von Kunstwerken ist Gegenstand eines Die Gemäblde überschriebenen Dialoges, den August Wilhelm Schlegel und seine Frau Caroline r799 veröffentlichten'. Schauplatz des fiktiven Gesprächs ist zunächst die Dresdner Galerie. Ein Maler, namens Reinhold, hat soeben versucht, eine antike Ringerfigur zu zeichnen, als zwei weitere befreundete Besucher, Louise und ein Dichter, namens Waller, zu ihm treten. Reinhold ist mit dem Ergebnis seiner Nachzeichnung unzufrieden und ruft - wohl etwas spöttisch - dem Freund zu:

*Reinhold: Nun, Waller, zeichnen Sie mir doch einmal den verwünschten Ringer da mit Worten $\mathrm{ab}$, da ich schon mit meiner Kreide so sehr den Kürzeren gegen ihn ziehe.
\end{abstract}

Waller: Sie verstehen mich unrecht, bester Freund. Es fällt mir nicht ein, mit der Sprache eben das ausrichten zu wollen, was nur ein sinnlicher Abdruck leisten kann. Ich sage bloß, daß sie fähig ist, den Geist eines Werkes der bildenden Kunst lebendig zu fassen und darzustellen.

Reinhold: Dieser so genannte Geist ist immer nicht die Sache selbst [...]

Louise: Lieber starrsinniger Reinhold, wie Sie sich dagegen setzen, daß man Statuen und Gemählde, die für sich ewig stumm sind, auch einmal

‡ Dieser Aufsatz ist die stark überarbeitete Fassung eines Vortrages, der im November r994 in Basel anläßlich einer Fachtagung der Jacob Burckhardt-Stiftung und des Graduiertenkollegs Die Renaissance in Italien und ibre europäische Rezeption (Universität Bonn) gehalten wurde. Anregungen in der anschließenden Diskussion verdanke ich insbesondere Bernd Roeck und Wilhelm Schlink. Den Anstoß zur Auscinandersetzung mit dem Thema der Kunstbeschreibung gaben mir Gottfried Boehm und Bernhard Schütz, weitere Anstöße gewann ich aus der Teilnahme an einem interdisziplinären Oberseminar zur Ekphrasis in Basel. Hilfreiche Hinweise habe ich von Esther Janowitz, Thomas Lersch, Martina Sitt, Christine Tauber und Wolf Tegethoff erhalten. Zu besonderem Dank für die kritische Durchsicht des Manuskriptes und für intensive Gespräche bin ich Gerd Blum, Huberta von la Chevallerie, Hans- reden lehren will! Wie soll man sich denn mit ihnen beschäftigen? $\times^{2}$

Auf diese Frage geben die drei Freunde unterschiedliche Antworten? ${ }^{3}$. Sie verkörpern darin verschiedene historische Traditionen: Louise hat ihrer Schwester Amalie versprochen, ihr *etwas von meinem hiesigen Genusse mitzubringen $*^{4}$ die Eindrücke der gesehenen Bilder für sie schriftlich festzuhalten. Dafür $* m u ß$ ich [Louise] mich an den inneren Menschen wenden, wenn ich seine Einbildungskraft interessieren will, ein noch nicht gesehenes Kunstwerk in sich zu erschaffen ${ }^{5}$. Im Gesprächsverlauf trägt sie beiden Männern mehrere Beschreibungen von Gemälden der Dresdener Sammlung vor. Weitere Beschreibungen steuert Waller bei. Diese Schriftstücke sind kritischer Natur, Beschreibung geht in Beurteilung über. Waller versteht sich allerdings weniger als Kunstkritiker denn als Dichter. Sein eigentliches Ziel ist *die Verwandlung von Gemählden in Gedichte ${ }^{6}$ und am Ende des Textes trägt er den Freunden einige dieser Bildgedichte vor. Reinhold, der Maler, möchte sich den Werken der bildenden Kunst am liebsten nur mit Zeichenstift und Pinsel nähern. Im Verlauf des Dialogs verteidigt er die Anwendung einer Fachsprache ${ }^{7}$ und lehnt die wertenden Beschreibungen Louises und Wallers

Jakob Meier, Ilse von zur Mühlen und meiner Frau Heidrun verpflichtet.

${ }^{1}$ Athenäum. Eine Zeitschrift von August Wilhelm und Friedrich Schlegel, II.I, 1799, zit. nach Reprint, Darmstadt $1983,39-15$ I.

${ }^{2}$ Athenäum (wie Anm. I), $48 \mathrm{ff}$.

${ }^{3}$ Gabriele Bickendorf: Der Beginn der Kunstgeschichtsschreibung unter dem Paradigma *Geschichte*. Gustav Friedrich Waagens Frühschrift * Uber Hubert und Johann van Eyck*, Worms $1985,46-54$ hat bislang am deutlichsten auf die unterschiedlichen Positionen der drei Gesprächspartner hingewiesen.

4 Athenäum (wie Anm. I), 5I.

sibid. 94 .

${ }^{6}$ Ibid. 137.

7 . Louse: Sic können sich leicht vorstellen, daß ich nicht 
$a b^{8}$. Er beteiligt sich jedoch an der von Louise im Gespräch entwickelten Schilderung der Sixtinischen Madonna Raffaels:

- Louise: Eine Göttin kann ich die Maria nicht nennen. Das Kind, was sie trägt, ist ein Gott, denn so hat noch niemals ein Kind ausgesehen. Sie hingegen ist nur das Höchste von menschlicher Bildung, und nimmt ihre Verklärung daher, daß sie den Sohn so still, so ohne sichtbare Regung von Entzücken oder Selbstgefühl auf ihren Arm hält, ohne Stolz und ohne Demuth? [...]

Sagen Sie, Reinhold, ist nicht das ganze Bild wie ein Tempel gebaut? Die beyden Figuren, welche rechts und links knieen, machen mit dem Schwunge der mittleren eine recht architektonische Symmetrie.

Reinhold: Sie nehmen sich wirklich in einiger Entfernung wie zwey Dreiecke aus, die ein schmales Oval zwischen sich tragen. Sie sind vor der Jungfrau einander so nahe gegenüber, daß ihr Gewand sie eben zu berühren scheint. Die Köpfe stehen ungefähr der Mitte der Hauptgestalt gleich. Die drey Figuren zusammen bilden wieder ein größeres Dreyeck, welchem oben ein von beyden Seiten schräg weggezogener grüner Vorhang parallel läuft. Alle diese Verhältnisse werden durch die hart gegeneinander abgeschnittnen Farben noch auffallender gemacht. Am härtesten steht das dunkelblaue Gewand der Madonna auf dem ganz weißen Grunde, der nur gegen seine äußere Gränze zu, wo die Engelsköpfe der Glorie kaum sichtbar angedeutet sind, bläulich wird; der schwere goldgewirkte Mantel des heiligen Sixtus und der graue Rock der Barbara, mit ihrer übrigen ziemlich bunten Tracht, zeichnen sich doch weniger stark aus. Die beyden Heiligen sinken tiefer in die

in Gefahr war, durch den Gebrauch der privilegierten Kunstwörter Amalien unverständlich zu werden. Es erschallt hier zwar genug um mich her von impasto, von Halbtinten, von Karnation, von Pyramidalgruppen, von Kontrapost, von beaux accidens de lumiere und so weiter, daß ich wohl einige dieser Ausdrücke hätte erhaschen können: aber mir ist, als würde mir durch sie das wieder verdunkelt, was ich an sich klar genug erkenne. $\alpha[. .$.

$\rightarrow$ Reinhold: Jedem Handwerke wird ja seine besondre Sprache vergönnt. Es sind doch nützliche Abbreviatu-
Wolken, und heben dadurch die Jungfrau; auch der Schatten unter ihren Füßen trägt zu ihrer hohen Leichtigkeit bey. [...]

Louise: Die junge Heilige, die so innig und anmuthig die Hände auf der Brust zusammenfaltet, wendet ihr Gesicht mit gesenktem Blick von der Madonna weg, nach ihrer vorderen Schulter herum. Sie ist zu schüchtern, um hinaufzuschauen, zu demüthig und auch mehr mit sich selbst beschäftigt $\alpha^{10}$.

Louises Beschreibungen nehmen emotionalen Anteil an der im Bild dargestellten Szene. Sie erzählen und kommentieren Haltung, Ausdruck und Handlung der Figuren. Aus einem Brief $\mathrm{Au}$ gust Wilhelm Schlegels an Goethe geht hervor, daß sie im wesentlichen von Caroline verfaßt wurden: ${ }^{2}$ a Sie doch wahrscheinlich die weibliche Hand darin erkennen würden, so darf ich thnen um so eher anvertrauen, daß meine Frau Theil daran hat. Die meisten Gemählde-Beschreibungen $[. .$.$] sind von ihr; sie bittet daher um Nach-$ sicht, wenn Sie es allzu poetisch und zu wenig artistisch finden sollten ${ }^{11}$. Wie zu zeigen sein wird, sind Louises bzw. Carolines Beschreibungen nicht so sehr das Ergebnis weiblicher Ästhetik als eine romantische Fassung der seit der Antike geläufigen Ekphrasis ${ }^{12}$. Diese Tradition soll im ersten Abschnitt umrissen werden. Demgegenüber steht der Maler Reinhold für eine sachliche und analytische, »artistische*, also fachmännische Bildbeschreibung. Sie ist uns inzwischen aus der wissenschaftlichen Kunstgeschichte vertraut. Ziel des vorliegenden Aufsatzes und Gegenstand der beiden folgenden Abschnitte ist es, die Geschichte dieser zweiten Position nachzuzeichnen und sie von der älteren Tradition der literarischen Ek-

ren, womit man sich am geschwindesten verständigen kann. * Athenäum (wie Anm. I), sif. vgl. auch 94.

${ }^{8}$ Ibid. 69, 120.

9 Ibid. 126.

ro Ibid. 13rf.

"Brief vom 8. 3. 1799, der die Lieferung der Zeitschrift an Goethe begleitete. Zit. nach Josef Körner und Ernst Wienecke (Hg.): A. W. und F. Schlegel im Briefwechsel mit Schiller und Goethe, Leipzig o. J., 84 f.

${ }^{12} \mathrm{Am}$ deutlichsten ist dies bei ihren Beschreibungen von Historiengemälden: Athenäum (wie Anm. 1), 76-93. 
phrasis abzugrenzen ${ }^{13}$. Dabei werde ich mich einfachheitshalber auf die sBildkünste, Malerei und Bildhauerei, beschränken. Schlegels dritter $\mathrm{Ge}$ sprächspartner, Waller, mit seiner Suche nach dem *Geist « der Werke, seiner Ablehnung der Beschreibung von Äußerlichkeiten und seinen Bildgedichten verkörpert eine typisch romantische Position, auf die in diesem Rahmen nicht weiter eingegangen wird ${ }^{4}$.

\section{Die antike Ekphrasis und ibr neuzeitliches Erbe}

Homers Beschreibung von Achilles' Schild ${ }^{55}$ ist nicht nur die älteste Ekphrasis der griechischen Literatur, sondern auch der in der Antike anerkannte Maßstab dieser Gattung ${ }^{16}$. Am Ende des XVIII. Gesangs der Ilias wird berichtet, wie Thetis Hephaistos besucht und ihn bittet, eine Rüstung für ihren Sohn zu schmieden und wie dieser sich ans Werk macht. Der Dichter verweilt bei der Herstellung des Schildes und bespricht die auf ihm kunstvoll gestalteten Bilder, indem er vor allem die dargestellten Handlungen erläutert. An Stelle einer Gegenstandsbeschreibung beinhaltet der Text damit zwei ineinander verwobene Erzählebenen: die der Entstehung des Werkes und jene der abgebildeten Szenen. Die Kunst des blinden

${ }^{13}$ Ekphrasen und Bildgedichte sind aufgrund ihres literarischen Gehaltes häufig Gegenstand wissenschaftlicher Untersuchungen gewesen. Die Erforschung sachlicher Bildbeschreibungen besitzt dagcgen den Charakter ciner historischen Hilfswissenschaft. Sie klärt uns über die Geschichte einer der wichtigsten Quellen und Medien der Kunstgeschichte auf. Trotz einiger Fallstudien fehlt bislang eine Geschichte der Bildbeschreibung. Besondere Beachtung verdient ein kürzlich erschienenes Gemeinschaftswerk, das sich dem Themenkomplex EkphrasisKunstbeschreibung erstmals in diesem Umfang widmet: Gottfried Boehm und Helmut Pfotenhauer ( $\mathrm{Hg}$.): Beschreibungskunst - Kunstbeschreibung. Ekphrasis von der Antike bis zur Gegenwart, München I995, mit umfangreicher Literaturliste $\left(6_{33}-642\right)$.

${ }^{4}$ Allgemein dazu Rosine Calsow: Die Methode der frühromantischen Bildkunstkritik, Berlin 1927 und August Langen: Ein Vorwort, in: W. H. Wackenroder: Herzensergießungen eines kunstliebenden Klosterbruders, mit einer Einführung von August Langen, Kempen 1948, bes. 20-24. Uber Wallers Gedichte siehe Karl Pestalozzi: Das Bildgedicht, in: Boehm u. Pfotenhauer (wie Anm. 13), 572-575.
Dichters ist es, durch diese Erzählungen intensive Bilder vor dem inneren Auge seiner Zuhörer zu evozieren. Dies ist paradoxerweise charakteristisch für alle Texte, die von der antiken Rhetorik als Ekphrasen bezeichnet werden, obwohl dort zugleich ,Ekphrasis als >Beschreibung definiert wird ${ }^{17}$. Der Praxis nach wäre die korrekte Übersetzung von ×Ekphrasis Bildererzählung und nicht Bildbeschreibung ${ }^{18}$.

In dieser Tradition verfaßt Philostrat ein Jahrtausend nach Homer seine Eikones. Zum ersten Mal sind dort die Beschreibungen von Gemälden nicht nur Teile eines literarischen Werkes, sondern sein ausdrücklicher Inhalt. Damit avanciert die $>$ Ekphrasis‘, die ursprünglich als Beschreibung eines beliebigen Gegenstandes definiert wurde und einen untergeordneten Teil der Rede bilden sollte, zur selbständigen literarischen Gattung. Mit dem Erfolg der Eikones beginnt offensichtlich auch die erst in der Neuzeit endgültig vollzogene Eingrenzung des Begriffes ,Ekphrasis a auf Beschreibungen von Kunstwerken ${ }^{19}$. Philostrats Schrift hat von Anfang an eine breite Rezeption erfahren. Sie hat viele spätantike und byzantinische Schriftsteller zur Nachahmung angeregt ${ }^{20}$.

Die antike Bilderzählung findet seit der Renaissance eine bedeutende Nachfolge. Svetlana Alpers

${ }^{15}$ Ilias 18, 478-608. Hierzu zuletzt Erika Simon: Der Schild des Achilleus, in: Bochm u. Pfotenhauer (wie Anm. 13), 123-I4I.

${ }^{16}$ Fritz Graf: Ekphrasis. Die Entstehung der Gattung in der Antike, in: Boehm u. Pfotenhauer (wie Anm. 13), I49ff.

17 Solche Definitionen sind seit dem I. Jh. n. Chr. in Rhetorik-Traktaten nachweisbar: ibid. 144-149.

${ }^{18}$ Über das Phänomen der Bilderzählung im allgemeinen siehe Thomas Frangenberg: Der Betrachter. Studien zur florentinischen Kunstliteratur des I6. Jahrhunderts, Berlin 1990, 67f.; Helmut Pfotenhauer: Winckelmann und Heinse. Die Typen der Beschreibungskunst im 18. Jahrhundert oder die Geburt der neueren Kunstgeschichte, in: Boehm u. Pfotenhauer (wie Anm. 13), 313328.

${ }^{19}$ Graf (wie Anm. 16), 143-155.

${ }^{20}$ Paul Friedländer: Johannes von Gaza und Paulus Silentiarius. Kunstbeschreibungen justinianischer Zeit, Leipzig und Berlin r 1912 . Die Einleitung ( $\left.\mathrm{x}-\mathrm{IO}_{3}\right)$ enthält die bislang ausführlichste Darstellung der * Beschreibung von Kunstwerken in der antiken Literatur $\alpha$. 
hat darauf hingewiesen, daß der größte Teil von Vasaris Vite aus Ekphrasen besteht ${ }^{21}$. $\gg$ Admitting their rhetorical character, we can $[\ldots]$ define the descriptions [In Vasaris Vite] as a revival and continuation of the traditional device of ekphrasis «. Alpers stellt dabei fest, daß Vasari Bilder ausschließlich im Hinblick auf narrative Eigenschaften beschreibt ${ }^{22}$, daß er sich für den dargestellten Stoff und hauptsächlich für den psychologischen Ausdruck der dargestellten Figuren interessiert ${ }^{23}$ und daß dies auch die typische Beschreibungsform in der Renaissance, namentlich bei Alberti, Ghiberti und Leonardo gewesen ist ${ }^{24}$. Vergleichbar narrativ und an szenischen Vorgängen orientiert sind auch viele Texte aus Diderots Salonkritiken $^{25}$. In seinem Salon von 1767 spricht er in einem imaginären Gespräch einen trivialen Literaten an: »Tu aurais dit d'un de tes combattants, qu'il avait reçu à la tête ou au col, une énorme blessure. $\star$ Der Dichter aber, als welcher sich Diderot versteht, sagt vom gleichen (gemalten) Krieger: sla flèche l'atteignit au-dessus de l'oreille, entra, traversa les os du palais, brisa les dents de la mâchoire inférieure, sortit par la bouche, et le sang qui coulait le long de son fer, tombait à terre en distillant par la pointe $^{26_{\alpha}}$. Es ist kein Zufall, daß diese epische Schilderung einer Kriegshandlung der Ilias entnommen zu sein scheint. Auch die frühen Be-

"Svetlana L. Alpers: Ekphrasis and aesthetic attitudes in Vasari's Lives, in: Journal of the Warburg and Courtauld Institutes, 23, 1960, 190 .

ax Ibid. IgI.

${ }^{23}$ Ibid. 193.

u Ibid. rg9f. Thomas Ketelsen: Künstlerviten. Inventare. Kataloge. Drei Studien zur Geschichte der kunsthistorischen Praxis, Ammersbeck 1990, 22-30 kritisiert Alpers' Vasari-Interpretation. Mit Recht betont er dic großen Unterschiede zwischen den Vite und Philostrats Eikones. Der urteilende Charakter der Schilderungen Vasaris widerspricht jedoch nicht deren grundsätzlich ekphrastischen Form.

${ }_{2}$ Vgl. August Langen: Die Technik der Bildbeschreibung in Diderots Salons, in: Romanische Forschungen, 6I, 1948, bes. $374-382$; Roland Virolle: Diderot: L.a critique d'art comme création romanesque dans les Salons (1765-1767), in: La critique artistique un genre littéraire, Paris 1983; Else Marie Bukdahl: Diderot critique d'art, I, Copenhague 1980 , $301-320$ unterscheidet die Beschreibungen nach »méthodes poétiques* von denen nach -méthodes scientifiques $\propto$. schreibungen Winckelmanns ${ }^{27}$ sowie der beschreibende Teil von Goethes Aufsatz über Leonardos Abendmahl sind der Tradition der Ekphrasis verpflichtet. Noch in der ersten Hälfte des 19. Jahrhunderts bleibt dieser Modus weit verbreitet ${ }^{28}$.

In einem 1788 erschienen Aufsatz mit dem Titel Inwiefern Kunstwerke beschrieben werden können schreibt Karl Philipp Moritz: »es ist offenbar, daß wir uns bei der Dichtung die Sachen um der Beschreibung willen, bei der Geschichte hingegen, die Beschreibung um der Sachen willen denken $\alpha^{22}$. In diesem Sinne sind die Ekphrasen von Homer, Philostrat und den meisten antiken Autoren um der Beschreibung und nicht um der Sache willen verfaß $\mathrm{t}^{30}$. Achilles' Schild aus der mythischen Schmiede des Hephaistos hat es außerhalb des Epos nie gegeben und die zahlreichen archäologischen Versuche seiner Rekonstruktion scheiterten sowohl an ausgegrabenen Vergleichsbeispielen, wie am Mangel konkreter Angaben ${ }^{31}$. Ähnlich fruchtlos erscheint der bereits jahrhundertealte Streit um die tatsächliche Existenz der von Philostrat geschilderten Bilder ${ }^{32}$. Der wandernde Rhetor ist um »belebte Beschreibungen $*$ bemüht ${ }^{33}$. Wie Homer verwandelt er statische Bilder in lebensvolle Handlung und verwischt die Konturen zwischen dem gemalten Werk und dessen

${ }^{26}$ Denis Diderot: CEuvres Complètes, éd. H. DieckmannJ. Varloot, XVI, Paris 1990, 286.

2) Pfotenhauer (wic Anm. 18), 3rfff.

${ }^{28}$ Regine Timm: Kunstbeschreibung und Illustration in Deutschland im 19. Jahrhundert, in: Peter Ganz u. a. $\left(\mathrm{Hg}_{\mathrm{g}}\right)$ : Kunst und Kunstrheorie $1400-1900$, Wiesbaden 1991, 34off.

${ }^{29}$ Karl Philipp Moritz: In wie fern Kunstwerke beschrieben werden kōnnen?, in: Monats-Schrift der Akademie der Künste und mechanischen Wissenschaften zu Berlin, I.2, 1788, zit. nach K. P. Moritz: Beiträge zur Ästhetik, hg. u. komm. v, Hans Jochim Schrimpf und Hans Adler, Mainz $1989,89$.

${ }_{30} \mathrm{Vgl}$. Graf (wie Anm. 16), 153.

3r Simon (wie Anm. Is), bes. 130.

${ }^{32}$ Dic bisherigen Positionen zusammengefaßt in: Philostratos: Die Bilder. Hg., übers. und erl. von O. Schönberger, München 1968, 26-37 und ders.: Die Bilder des Philostratos, in: Boehm u. Pfotenhauer (wie Anm. 13), I62f. Schönberger selber ergreift Partei für jene, die an der realen Existenz der Vorbilder glauben ( $163 f_{\text {. }}$ ).

${ }^{33}$ Ibid. 158. 
literarischen Vorlage. Diese Ekphrasen erfüllen die Einbildungskraft ihrer Leser noch heute mit farbigen Bildern, deren Leben allerdings keinen Rahmen kennt. Ihr Ziel ist nicht die Vergegenwärtigung von spezifischen Bildwerken, sondern die Schöpfung eines literarischen Werkes.

Anders als bei Homer oder Philostrat besteht allerdings bei Vasari, Diderot, Winckelmann und Goethe kein Zweifel an der Existenz der von ihnen beschriebenen Werke. Der konkrete Bezug dieser Ekphrasen auf die »Sache selbst« (Reinhold), auf die spezifische Gestalt des jeweiligen Werkes, nimmt im Laufe der Zeit deutlich zu. So schreibt beispielsweise Goethe: »Petrus hat indessen mit seiner linken Hand des gegen ihn geneigten Johannes rechte Schulter gefaßt, hindeutend auf Christum, und zugleich den geliebten Jünger anregend, er solle fragen, wer denn der Verräter sei? Einen Messergriff in der Rechten, setzt er dem Judas unwillkürlich zufällig in die Rippen, wodurch dessen erschrockene Vorwärtsbewegung, die sogar ein Salzfaß umschüttet, glücklich bewirkt wird $\star^{34}$. Seit der Antike hat sich ein grundlegender Wandel des Interesses vollzogen: von der Be-

\section{${ }^{34}$ Johann W. Goethe: Giuseppe Bossi: Úber Leonardo da Vincis Abendmahl zu Mailand, zuerst in: Uber Kunst und Altertum, I. 3, 1817, zit. nach Goetbes Gedenkaus- gabe, XIII, Zürich ${ }^{2} 1965,750$.}

3s Die Dresdner Sammlungsgeschichte bietet sich als Beispiel an (vgl. Gerald Heres: Dresdner Kunstsammlungen im 18. Jahrhundert, Leipzig $199 \mathrm{r}$ und Harald Marx u. Heinrich Magirius: Gemäldegalerie Dresden. Die Sammlung alter Meister. Der Bau Gottfried Sempers, Leipzig 1992). Als das Ehepaar Caroline und August Wilhelm gemeinsam mit dem Bruder Friedrich Schlegel, mit Fichte, Schelling, Novalis und anderen sich im Juli 1799 der Betrachtung der Königlichen Sammlung widmeten, fanden sie eine damals gerade fünfzig Jahre alte Hängung in dem nach Plänen Knöffels neugebauten *Stallgebäude * $(I 745-46)$ vor. Italienische und nordalpine Bilder waren in zwei, um einen Hof konzentrisch umlaufenden Galerien getrennt ausgestellt. Die Bilder tapezierten deckenhoch, dicht an dicht in drei bis vier Lagen übereinander gehängt, die Ausstellungswand (vgl. die Ansicht der inneren Galerie von 1830 , abgedruckt bei Heres, I2I und bei Marx u. Magirius, II). Der junge Goethe hatte die Sammlung 1768 besucht und schreibt in Dichtung und Wabrbeit: - Ich trat in dieses Heiligtum, und meine Verwunderung überstieg jeden Begriff, den ich mir gemacht hatte. Dieser in sich selbst wiederkehrende Saal, in welchem Pracht und Reinlichkeit bei der größten Stille herrschten, die blendenden schreibung zur beschriebenen Sache. Die Sprache ist zunehmend der Treue zum Werk verpflichtet. Die wachsende Berücksichtigung bestimmter Werke in ihrer je eigenen Gestalt steht im Zusammenhang mit der allgemeinen Aufwertung des Kunstwerkes. Stufen dieser Aufwertung in der Kunstliteratur sind die Trennung von Leben und Werk innerhalb der Künstlermonographien (erstmals in Belloris Vite, 1672) wie die Verfassung von künstlerunabhängigen Geschichten von Kunstwerken (ausgehend von Winckelmanns Geschichte der Kunst des Altertums, 1764). Seit dem ausgehenden Cinquecento entstehen die ersten Schriften, die einem einzelnen Werk der bildenden Künste gewidmet sind. Die Anzahl monographischer Aufsätze und Bücher scheint vor allem nach der Mitte des I9. Jahrhunderts schnell zu steigen. Die Aufwertung des Einzelwerkes ließe sich wahrscheinlich auch an einer Geschichte der Kunstmarktpreise verfolgen. Sie spiegelt sich jedenfalls in der Entwicklung der musealen Ausstellungspraxis von den nach dekorativen Gesichtspunkten »tapezierten « Galeriewänden hin zu einer das Einzelwerk in Szene setzenden Hängung35.

Rahmen, alle der Zeit noch näher, in der sie vergoldet wurden, der gebohnte Fußboden, die mehr von Schauenden betretenen als von Arbeitenden benutzten Räume gaben ein Gefühl von Feierlichkeit, einzig in seiner

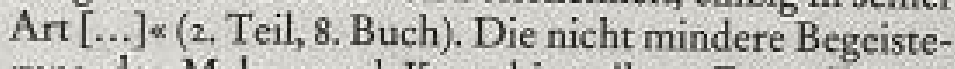
rung des Malers und Kunsthistorikers Ernst Förster aus seinen Briefen von 1838 , zeigt einen deutlich gewandelten Zugang zur gleichen Sammlung. So liest man beispielsweise: $*$ Wunderbarer Eindruck, wenn $\mathrm{Du}$, ohne Neues in Dein' Auge zu lassen, von Tizians Venus zur Sixtina gehst. « (Ernst Förster: Briefe über Malerei, Stuttgart u. Tübingen 1838, 92). Nicht mehr der Gesamteindruck des weihevollen Kunstraumes, sondern ausschließlich einzelne Meisterwerke stehen für Förster im Mittelpunkt des Interesses, und die 1855 erfolgte Neuaufstellung der Gemälde im Semperbau, in dem die Sixtina in einem eigens für sie eingerichteten Raum aufgestellt ist, trägt diesem Rechnung: das Bild erhalt einen altarähnlichen Rahmen, wobei an Stelle eines Antependiums Vasaris Satz über das Bild gemeißelt ist. Die Besucher konnten sich nun auf mit rotem Samt bezogenen Stühlen in die Betrachtung von Raphaels Meisterwerk vertiefen. Selten war die Grenze zwischen religiöser Kunst und Kunstreligion so unscheinbar (vgl. Michael Ladwein: Raphaels Sixtinische Madonna: Zeugnisse aus zwei Jahrhunderten deutschen Geisteslebens, Stuttgart 1993, 80. Eine Abbildung dieser Ausstellungssituation bei Marx u. Magirius, 18). 


\section{Die Entstehung der sachlichen Bildbeschrei- bung}

Die Bemühung um das einzelne Werk, um die

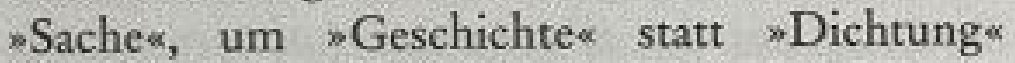
kommt vor allem in einer zweiten Tradition der Beschreibung zum Tragen, deren Anfänge, im Schatten der dichterischen Bilderzählungen, nur selten Beachtung gefunden haben. Es handelt sich um Texte, bei denen die Evokation lebendiger Bilder weniger zählt als die sachliche Schilderung eines bestimmten Bildwerkes, seiner individuellen Genese, seiner spezifischen, optischen Gestalt und seiner besonderen Wirkung ${ }^{36}$.

Paul Friedländer findet den Ursprung solcher Kunstbeschreibungen in der Geschichtsschreibung Herodots ${ }^{37}$. Schönberger verfolgt die Wurzeln der "sachlichen Schilderungen « sogar bis Hesiod zurück ${ }^{38}$. Die am besten überlieferten und bekanntesten antiken Texte dieser Art befinden sich in Pausanias Beschreibung Griechenlands ${ }^{39}$. Im Gegensatz zu Homer oder Philostrat gibt es keinen Zweifel über die Realität der von ihm geschilderten, teils ausgegrabenen Denkmäler. Seine Beschreibungen bestehen aus informativen, meist rein ikonographischen Benennungen der dargestellten Figuren. Als Beispiel seien wenige Zeilen einer langen Passage über die Lade des Kypselos $(\mathrm{V}, 17,5-19, \mathrm{I0})$ zitiert: $\bowtie$ Beginnt man mit der Betrachtung unten, so enthält der erste Streifen auf

${ }^{56}$ In viel höherem $\mathrm{Maß}$ als bei der Ekphrasis stellt sich bei der Bildbeschreibung das Problem des Verhältnisses zweier verschiedener Medien, das Problem, eine sprachliche Äquivalenz für Bildliches zu finden. Von den neueren Beiträgen hierzu seien folgende hervorgehoben: Michael Baxandall: The Language of Art History, in: New Literary History, 10, 1979, 453-465; Gottfried Boehm: Bildbeschreibung. Uber die Grenzen von Bild und Sprache, in: Bochm u. Pfotenhauer (wic Anm. 13), 23-40; Emil Angehrn: Beschreibung zwischen Abbild und Schöpfung, ibid., 59-74 und Brigitte Hilmer: Kunstphilosophische Überlegungen zu einer Kritik der Beschreibung, ibid, $75-97$. Siche auch die prägnante Definition von sDescrizione in Salvatore Battaglias Grande dizionario della lingua italiana: sdiscorso mediante il quale una cosa viene individuata, dichiarandone i caratteri accidentali, che la distinguono da un'altra (e differisce dalla definizione, in quanto questa dichiara l'essenza) * (VI, 1966, 238).

${ }_{77}$ Friedländer (wie Anm. 20), 32. der Lade folgendes. Oinomaos ist dargestellt, wie er Pelops verfolgt, der Hippodameia bei sich hat; beide von ihnen haben zwei Pferde, und die des Pelops sind sogar geflügelt. Weiter ist das Haus des Amphiaraos abgebildet, und eine alte Frau, wer es auch sein mag, trägt den unmündigen Amphilochos. Vor dem Haus steht Eriphyle mit dem Halsband und neben ihr ihre Tochter Eurydike und Demonassa und der Knabe Alkmaion nackt $\alpha^{40}$. Im aufzählenden Charakter seiner Beschreibung scheint sich eine friesartige Anordnung des Werkes niederzuschlagen. Der Kontrast zu einer der Philostratischen Ekphrasen mit gleichem Thema (I, 17,2 f.) könnte kaum größer sein: "Sieh nur die Rosse des Oinomaos, wie gewaltig sie sind und vorwärts stürmen wollen, wie hitzig und mit Schaum bedeckt und wie schwarz sie sind!... Im Wettrennen also haben Pelops und Hippodameia gesiegt; sie stehen beide auf dem Wagen, Hand in Hand und so voneinander beseligt, daß sie sich gleich umarmen möchten, er in der Blüte und Schönheit der Jugend, sie geschmückt wie eine Braut $\alpha^{41}$.

Die prosaische Tradition der Bildbeschreibung gewinnt seit der Renaissance zunehmend an Bedeutung. Neben den zahlreichen Ekphrasen beinhalten Vasaris Vite - insbesondere jene des Michelangelo - auch ausführliche Beschreibungen, die auf die spezifische visuelle Gestalt bestimmter Werke eingehen ${ }^{42}$. Als Beispiel sei die Beschrei-

38 Schönberger: Die Bilder des Philostratos (wie Anm. 32), $158 f$.

39 Eine Liste der cinschlägigen Stellen bei Salvatore Settis: La trattatistica delle arti figurative, in: Lo spazio letterario della Grecia antica, I (La produzione e la circolazione del testo), 2 (L'Ellenismo), Roma 1993, 482.

$4^{\circ}$ Zit. nach der Ubersetzung von Ernst Meyer, Zürich $1954,268 \mathrm{f}$.

4'Zit. nach Schönberger: Dic Bilder des Philostratos (wie Anm. 32), 159 .

${ }^{42}$ Neuzeitliche Vorläufer Vasaris sind möglicherweise in den Beschreibungen antiker Statuen der ersten Hälfte des Cinquecento zu finden. Ein Beispiel ist die ungewöhnlich detaillierte Schilderung des Laokoon, die die venetianischen Gesandten in dem Bericht einer Audienz Hadrians VI (1523) geben. Abgedruckt in: Eugenio d'Alberi: Relazione degli Ambasciatori Veneti al Senato, Ser. II, Tomo III, Firenze 1876 , 115 f. Diesen Hinweis verdanke ich Michael Waltz. 
bung des Propheten Jeremias aus der Sixtinischen Decke zitiert: Vedesi quel Ieremia con le gambe incrocicchiate tenersi una mano alla barba, posando il gomito sopra il ginocchio, l'altra posar nel grembo et aver la testa chinata d'una maniera che ben dimostra la malenconia, i pensieri, la cogitazione e l'amaritudine che egli ha del suo popolo; così medesimamente due putti che gli sono dietro ${ }^{43}$. Auffällig ist, daß Propheten und Sybillen eingehender beschrieben sind als die *istorie der Genesis an der Decke, ja überhaupt als alle andere Figuren der Kapelle. Gegenstand einer genauen Beschreibung ist bei Vasari nicht ein ,Bild im heutigen Verständnis, sondern stets eine oder mehrere dargestellte Figuren ${ }^{44}$. Vasari würdigt durch ausdrückliche Beschreibung die Figurengestaltung, ein zentrales Anliegen von Michelangelo, und zeigt das primäre Interesse, das er selbst an der Darstellung der menschlichen Figur hat. Hans Körner hat gezeigt, daß die oberste Stufe für die Begriffe der >Einheit und der Komposition in der Kunsttheorie der Renaissance die Komposition der Körper« war ${ }^{45}$, daß es also um die Mitte des I6. Jahrhunderts noch kaum eine diskursive Ebene gab, in der das Ganze eines Bildes, Bildformat, Gesamtkomposition, Hintergrund usf. zur Sprache kommen konnte ${ }^{46}$. Es überrascht nicht, daß Lodovico Dolce, in seinem Dialogo della Pittura, den Schlosser als venetianische Absage an

${ }^{43}$ Giorgio Vasari: La vita di Michelangelo nelle redazioni del 1550 e del is68. Curata e commentata da Paola Barocchi, Vol. I, Milano u. a. 1962, 46 .

${ }^{44}$ Außer von Propheten und Sybillen der Sixtinischen Decke (ibid. 46-49) gibr Vasari in der Vita Michelangelos detaillierte Beschreibungen des Kartons für die Scblache bei Cascina namentlich von dem Mann mit Efeukranz, der seine Socken anzieht (2sf.), der als Einzelstich durch Marcantonio Raimondi verbreitet worden war, der Madonna Medici (6of.) und - seit der zweiten Auflage von 1568 - des Tondo Doni. Die vieldiskutierte Beschreibung des Jüngsten Gerichts ( $76-80)$ ist angesichts der Größe und Figurenanzahl dieses Freskos verhältnismäßig kurz. In ihr verbindet Vasari beide Beschreibungsmodi. Einerseits wird er der spezifischen Gestaltung der Wand, die er von oben nach unten beschreibt, gerecht und andererseits erzäblt er dabei das Geschehen am Jüngsten Tag.

${ }^{45}$ Hans Körner: Auf der Suche nach der *wahren Einheit $\alpha$. Ganzheitsvorstellungen in der französischen
Vasaris Michelangelo-Kult bezeichnet hat ${ }^{47}$, in der Beschreibung von Tizians Assunta andere Schwerpunkte setzt: $»[\ldots]$ gli fu data a dipingere una gran tavola all'altare grande della chiesa de' Frati Minori, ove Tiziano, pur giovanetto, dipinse a olio la Vergine che ascende al cielo fra molti angioli che l'accompagnano, e di sopra lei affigurò un Dio Padre attorniato da due angioli. Par veramente che ella ascenda, con un volto pien d'umiltà, e il panno vola leggiadramente. Nel piano sono gli Apostoli, che con diverse attitudini dimostrano allegrezza e stupore, e sono per la maggior parte maggiori del vivo. E certo in questa tavola si contiene la grandezza e terribilità di Michelagnolo, la piacevolezza e venustà di Rafaello, et il colorito proprio della natura $\alpha^{48}$. Noch deutlicher als Vasari unterscheidet Dolce das Sujet des Bildes (*la Vergine che ascende al cielo*) von dessen Wirkung ( $*$ Par veramente che ella ascenda $\alpha$ - sie scheint also tatsäcblich gen Himmel zu fahren)*9. Seine Beschreibung spiegelt andererseits den klaren Bildaufbau Tizians wieder. Er unterscheidet drei Ebenen: Maria mit den vielen sie begleitenden Engeln, Gottvater von zwei Engeln umgeben, die Apostel auf der (unteren) Ebene. Dolce verliert sich in keiner Detailbeschreibung, hebt den markanten Mantel Mariens und ihren Gesichtsausdruck hervor, geht aber nicht auf die Haltung einzelner Figuren ein. Der Vergleich mit Michelangelo, Raf-

Malerei und Kunstliteratur vom mitteren 17. bis zum mittleren 19. Jahrhundert, München 1988, bes. 14-24.

${ }_{46}$ Vasaris Beschreibung von Michelangelos Jüngstem $\mathrm{Ge}$ richt kann als früher Versuch einer übergreifenden Bildbeschreibung gesehen werden. Sie befindet sich bereits in der ersten Auflage (vgl. Anm. 44). Wie wenig selbstverständlich dies ist, zeigt sich darin, daß Vasari einen Überblick der Gliederung der Decke der Sixtinischen Kapelle erst in der zweiten Auflage der ansonsten unveränderten Beschreibung ihrer einzelnen Teile voranstellt: Vasari (wie Anm. 43), 41, Zeile 15-25.

77 Julius von Schlosser: Die Kunstliteratur. Ein Handbuch zur Quellenkunde der neueren Kunstgeschichte, Wien 1924, 350.

${ }^{4}$ Lodovico Dolce: Dialogo della pittura intitolato I'Aretino (I557), zitiert nach Paola Barocchi $\left(\mathrm{Hg}_{\mathrm{g}}\right)$ : Trattati d'arte del Cinquecento, Bari 1960, I, 202.

49 Wie sehr das Gefälle von Tatsache und Wirkung das zentrale Moment einer Kunstbeschreibung ist, betont Boehm (wie Anm, 36), 3i-38. 
fael und der Natur, der aus heutiger Perspektive topisch erscheint, ist nicht nur lobend, sondern auch charakterisierend und also beschreibend zu verstehen. Man achte beispielsweise auf die kraftvolle, michelangeleske Gestaltung von Tizians Apostelfiguren.

Die ausführliche Beschreibung eines bestimmten Werkes der bildenden Kunst, seiner spezifischen Gestalt und Wirkung bleibt im 16. Jahrhundert eher die Ausnahme. Vasari wie auch Dolce setzen sie gezielt ein, um einige wenige Werke hervorzuheben. Dieses Verfahren eignet sich Francesco Bocchi an, indem er die Beschreibung der Kunstdenkmäler von Florenz zum Lob seiner Heimatstadt verwendet ${ }^{5 \circ}$. Le Bellezze della Città di Fiorenza (1591), »der erste Führer größeren Umfangs mit ausgesprochen kunsthistorischer Richtung ${ }^{\pi}$, beinhaltet die größte Sammlung ausführlicher Bildbeschreibungen des Cinquecento. Bocchi war Literat, Rhetor, und bezog seinen Lebensunterhalt aus der Erziehung adliger Zöglinge ${ }^{52}$. Indem er sich der bildenden Kunst widmet, ist er sich bewußt, Fremdland zu betreten und zitiert stets das Urteil der »artefici« und *intendenti $\alpha^{53}$. In der bereits 1571 verfaßten Monographie über die Eccellenza della statua di San Giorgio di Donatello kommt noch keine beschreibende Zeile vor ${ }^{54}$. Dadurch bleibt diese sälteste kunsthistorische Monographiesss vollkommen un-

${ }^{5 \circ}$ Francesco Bocchi: Le Bellezze della Citta di Fiorenza, Dove à pieno di Pittura, di Scultura, di sacri Tempij di palazzi i più notabili artifizij, \& più preziosi si contengono, Fiorenza 1591, zit. nach Reprint, 0.O. 1971. Siehe dazu John Shearmans Einleitung zu diesem Reprint und Frangenberg (wie Anm. 18), passim, bes. 130-147, der zum ersten Mal Bocchis ekphrastische Leistungen untersucht und würdigt.

"Schlosser (wie Anm. 47), 330.

52 S. Menchi: Francesco Bocchi, in: Dizionario Bibliografico degli Italiani, II, Roma 1969, 72-74.

${ }^{93}$ Ein Beispiel unter vielen anderen: $*$ [Michelangelo's] Crepuscolo, il quale fatto di stupendo artifizio, incredibilmente è da gli artefici ammirato. Et di vero cotanto è in ogni parte questa figura maravigliosa, che saziar non si puote chi più intende; e gli artefici, quando considerano attentamente nella bellezza oltra tutta sovrana restano confusi.* Bocchi: Bellezze (wie Anm. 50), 27 If. Robert Williams: A treatise by Francesco Bocchi in praise of Andrea del Sarto, in: Journal of the Warburg and Courtauld Institutes, 52, 1989, ngf. betont, wie schr spezifisch und könnte wortwörtlich für fast jede andere Gewandstatue der Renaissance übernommen werden. Die rein rhetorische Sprache, macht die beschränkte Rezeption dieser Schrift verständlich $^{56}$. Die späteren Bellezze verfolgten zwar ein ähnliches, panegyrisches Ziel wie die frühere Schrift. In den zwanzig Jahren, die beide trennen, hat jedoch eine auffällige Annäherung an die zu besprechenden Werke stattgefunden. Bocchi lobt die Florentiner Denkmäler mit repetitiven Formulierungen, die einer geringen Anzahl kunsttheoretischer Kategorien entsprechen: sei es die gelungene Mimesis, das angemessene Decorum oder die Facilità, mit der eine schwierige Aufgabe gemeistert wurde ${ }^{57}$. Dieses standardisierte Lob geht jedoch von je spezifischen, beschreibenden Feststellungen aus: »La gamba sinistra [des Giorno Michelangelos], che posa in sù la coscia, come in atto di natura sovente si vede, oltra ogni stima da gli artefici è ammirata $\alpha^{58}$. Stellt man die beschreibenden Teile seines Textes zusammen, fällt auf, »daß Bocchi seine Beschreibung meisterlich strukturiert und eine Fülle von subtilen Beobachtungen anstellt, die Gestalt und Wirkung [des Werkes] verständlich werden lassen ${ }^{59}$. Darin unterscheiden sich Bocchis Bellezze grundlegend von der Eccellenza, in der es bei der Erörterung allgemeiner theoretischen Kategorien und Antikenzitate blieb.

Bocchi ein Künstlerpublikum vor Augen hat. Vgl, auch Frangenberg (wie Anm. 18), 138.

"Eccellenza della statua del San Giorgio di Donatello Scultore Fiorentino, posto nella facciata di fuori d'Or San Michele, scritta da M. Francesco Bocchi in lingua fiorentina: dove si tratta del costume, della vivacità $e$ della bellezza di detta statua, Fiorenza 1584 (laut Widmung an Cosimo bereits is 71 verfaßt).

"Schlosser (wie Anm. 47), 348.

${ }_{56} \mathrm{Zur}$ Rezeption siehe die nota critica in der Ausgabe von Bocchis Eccellenza bei Paola Barocchi: Trattati d'Arte del Cinquecento, Bari 1962, III, 392f. Die Analyse der rhetorischen Voraussetzungen dieser Schrift ist auch Gegenstand einer unpublizierten Bochumer Magisterarbeit von G. Schröder.

77 Siehe beispielsweise die Beschreibung von Michelangelos Giomo in Bocchi: Bellezze (wie Anm. 5o), $266 \mathrm{ff}$. Uber Bocchis Urteilskriterien vgl. Williams (wie Anm. 53), III-I2I.

${ }_{58}^{8}$ Bocchi: Bellezze (wie Anm. 50), 267f.

${ }^{59}$ Frangenberg (wie Anm. 18), $138 f$. 
Im Herbst 1602 sah Monsignore Agucchi im Hause Farnese das großformatige Leinwandbild einer schlafenden Venus mit spielenden Amoretten von Annibale Carracci (heute in Chantilly) ${ }^{60} . \mathrm{Er}$ selber berichtet, wie ihm das Bild so gut gefiel, daß er das Bedürfnis hatte, eine Kopie (*ritratto*) davon mitzunehmen und sogleich begann, das Werk abzuzeichnen wà conservarne sempre viva la forma nella mia imaginazione ${ }^{61}$. Und obwohl das Zeichnen seinen Bedürfnissen und seinem Beruf als gelehrtem Kirchenmann fremd ist, bereute er bei dieser Gelegenheit zutiefst, es nie gelernt zu haben. Als er dann nach Hause kam, setzte er seine literarische Fähigkeiten ein, um dem fehlenden zeichnerischen Können abzuhelfen und beschloß zu beschreiben, was nachzuzeichnen ihm nicht vergönnt war $^{62}$. Den Angaben über Breite und Höhe der Leinwand ( 503 ) folgt eine zusammenfassende Inhaltsangabe und Charakterisierung des Bildes (503f.). Danach beschreibt Agucchi den landschaftlichen Hintergrund mit Blumen und Bäumen sowie das Bett, auf dem Venus ruht (504). Ausführlich und detailliert diskutiert der Kleriker die Schönheit ihres hüllenlosen Körpers, ihre Haltung, das auf sie fallende Licht und die Farbe ihres Inkarnats (504-507). Er fordert den Leser dann auf,

${ }^{6}$ Elisabeth de Boissard u. Valérie Lavergne-Durey: Chantilly, Musée Condé. Peintures de l'Ecole italienne, Paris 1988, Nr. 21, 67-69 mit Abbildung und Bibliographie.

${ }^{6}$ Monsignor Agucchi: Descrittione della Venere dormiente di Annibale Carrazzi, erstmalig abgedruckt in: Carlo Cesare Malvasia: La Felsina Pittrice, Vite de Pittori Bolognesi..., Bologna I678, I, 503 (in Malvasias Ausgabe von Gianpietro Zannotti, Bologna 1841, I, 360). Agucchi erwähnt die Descrittione in zwei Briefen vom April ${ }_{1603}$ (vgl. Anm. 64 und 65) und gibt an, sie zur Zeit der Weinlese verfaßt zu haben. Daraus ergibt sich die Datierung der Beschreibung und ein Datum ante quem für das Bild.

${ }^{62}$ risolsimi con troppo maggior mio gusto di supplire coll'industria della penna al mancamento dell'arte, e d'impiegarle nello descrivere in carta quello, che di raffigurare in altra guisa, non erami conceduto: e ne distesi perciò questa piacevole descrittione* Agucchi, Descrittione (wie Anm. 61), 503.

${ }^{63}$ Eugenio Battisti: L'Antirinascimento con un appendice di manoscritti inediti, Milano 1962,530 hat dies bereits gewürdigt: wa Descrizione della Venere dormiente di Annibale Carrazzi $[\ldots]$ segna veramente una svolta sein Auge von diesem vollendeten Körper zu trennen, um sich der Betrachtung der Amoretten - 28 an der Zahl - zuzuwenden. Der Monsignore beschreibt sie einzeln, ortet sie im Bild, schildert ihre Spiele und ihren Ausdruck (507-513). Der Text endet mit einem umfassenden Lob des Gemäldes (513f.). Neu ist bei Agucchi vor allem sein Anspruch, eine - wenn auch unvollkommene - Reproduktion des Werkes zu geben. Die Genauigkeit und Ausführlichkeit seiner Beschreibung ist für damalige Verhältnisse einzigartig und die logische Konsequenz dieses Anspruches ${ }^{63}$. Agucchis Beschreibung wurde in einem Kreis von Literaten und Kunstliebhabern verbreitet, abgeschrieben und diskutiert ${ }^{64}$. Ein solcher Liebhaber war sein Bologneser Freund Bartolomeo Dulcini. Er hatte Annibales römisches Venusbild noch nicht sehen können, als er Agucchis Descrittione erhielt. Dulcini bat darauf einen jungen Künstler, eine Zeichnung nach dieser Beschreibung anzufertigen und schickte sie Agucchi zu. Der Monsignore, Annibale und ihr römischer Kreis (»tutti gli altri staunten über diese Zeichnung sehr und waren von der Ähnlichkeit zum Urbild beeindruckt. Agucchi versprach schließlich, Dulcini eine Nachzeichnung von Carraccis Gemälde zukommen zu

nelle *letteratura artisticas: lo scrittore rivaleggia con il pittore, allo scopo di dare dell'immaggine una trascrizione ricca e aderente, sostitutiva quasi dell'originale. . Das Verständnis von Beschreibung als Ersatz einer Abbildung kommt schon früher auf, wird jedoch bei der Durchführung von Beschreibungen nicht eingelöst. Ascanio Condivi (Vita di Michelagnolo Buonarroti, Roma 1553, zitiert nach Guido Vitaletti: Michelangelo. Lettere e Rime. Precedute dalla Vita di Ascanio Condivi..., Torino 1942,96) schreibt im Bezug auf das Jüngste Gericht in der Sixtinischen Kapelle: $\gg$ La composizione della storia è prudente e ben pensata, ma lunga a descrivere e forse non necessaria, essendone stati stampati tanti e cosi vari ritratti e mandati per tutto.* Dieser Gedanke muß Vasari gefallen haben, so daß er ihn an der gleichen Stelle seiner Vita in der zweiten Auflage von 1568 übernimmt: *Nè verrò a' particolari della invenzione o componimento de questa storia, perchè se n'è ritratte e stampate tante, e grandi e piccole, che e' non par necessario perdervi tempo a descriverla. « (wie Anm. 43, 74).

${ }^{64} \mathrm{Vgl}$. Agucchis Brief vom 23. 4. 1603, in: Battisti (wic Anm. 63), 547. 
lassen ${ }^{65}$. Die Übersetzung von Bild in Sprache und Sprache in Bild sowie der Vergleich von Ähnlichkeiten und Unterschieden ist also zu einem Spiel im gelehrten Kreis geworden. In dieser Hinsicht ist Agucchis Schrift ein frühes Beispiel für das barocke Denken, das alle Welterscheinungen in logische, sprachliche Systeme übersetzt, für den von Michel Foucault diagnostizierten sDiskurs der Repräsentation $<$ des »âge classique ( 17 . und 18 . Jahrhundert) ${ }^{66}$.

Agucchi hat mit der Beschreibung von Carraccis Venus neue Horizonte der sprachlichen Annäherung an Bilder eröffnet. In seiner Nachfolge steht Ferrante Carlo, der zwischen 1627 und 1628 eine Descrittione della Cupola di S. Andrea della Valle depinta dal Cavalier Gio: Lanfranchi verfaßte ${ }^{67}$. Nicholas Turner, dem die Edition und Analyse dieses Textes zu verdanken ist, vermutet, daß Carlo das Programm der Kuppelfresken entworfen hat und weist auf die mögliche Rolle dieser Schrift im Kontext der Rivalität zwischen Lanfranco und Domenichino hin ${ }^{68}$. Carlo übernimmt von Agucchi den gattungsbezeichnenden Titel der $*$ Descrittione . Wie dieser vergleicht er Beschreiben und Nachzeichnen ${ }^{69}$ und schildert in geordneter Reihenfolge jede Figur auf Lanfrancos Kuppel, ihre Haltung und ihren Ausdruck ${ }^{70}$. Während Agucchis Text im wesentlichen einem reflektierten Gang durch das Gemälde glich, trennt Carlo die Besprechung von Architektur- und Figuren-

${ }^{6}$ Dies geht aus cinem Brief Agucchis an Dulcini vom April 1603 hervor. Siche Anton W. A. Boschloo: Due lettere inedite di Mons. Giovan Battista Agucchi in cui si parla di Ludovico, Agostino, e Annibale Carracci, in: L'Arte, 14, 1971, 71f. u. Anmerkungen.

${ }^{66}$ Michel Foucault: Les mots et le choses, Paris 1966. Foucault schreibt beispielsweise: le jeu des similitudes était autrefois [in der Renaissance] infini; il était toujours possible d'en découvrir de nouvelles [...]. Maintenant [seit dem 17. Jahrhundert] une énumération complète va devenir possible (69). Die Bildbeschreibung als solche thematisiert Foucault nicht. Er leitet jedoch seine Darstellung mit der Beschreibung eines Bildes ein (Velázquez' Meninas).

${ }^{67}$ Nicholas Turner: Ferrante Carlo's Descrittione della Cupola di S. Andrea della Valle depinta dal Cavalier Gio: Lanfranchi; a source for Bellori's descriptive method, in: Storia dell'Arte, 12, 1971, 297-325.

${ }^{68}$ Ibid. 299f. beschreibung, Stil und Ikonographie. Jeder Abschnitt ist mit einem Untertitel überschrieben: nach einigen einleitenden Sätzen (3isf.) beschreibt er zuerst sehr genau die Kuppel, in der sich das Fresko befindet (Cupola, 3i6f.), dann die einzelnen Figuren und ihr Verhältnis zueinander (Historia, 317-321). Darauf folgen Ausführungen über den Stil dieser Malerei (Maniera, 321-323). Zum Schluß diskutiert Carlo die ikonographische Erfindung des Kuppelprogramms (Inventione, 323f.). Carlo lobt in jeder dieser Kategorien das Werk und verteidigt Lanfranco gegenüber möglichen Vorwürfen. Die Beschreibung gewinnt dadurch den Charakter eines Gutachtens. Die in den Überschriften Carlos enthaltenen Kategorien lassen sich in der Kunstliteratur des Cinquecento zurückverfolgen. Auch Dolce hatte seiner Beschreibung der Assunta Bemerkungen über die maniera folgen lassen ${ }^{71}$. Carlo dürfte jedoch der erste sein, der die Beschreibung eines Kunstwerkes nach getrennten Rubriken konsequent und ausführlich durchführt. Er wendet damit eine Form der Werkanalyse an, die in der Kunstgeschichtsschreibung bis heute wichtig geblieben ist $^{72}$.

Der Kontext der Descrittioni von Agucchi und Carlo ist denkbar verschieden: gelehrte Mußs im einen, Erklärung, Lob und Verteidigung im Klima eines Künstlerstreites im anderen Fall ${ }^{73}$. Beide kamen erst posthum zum Druck. Sie waren Bellori

69 sittura da noi più tosto mirata di passaggio, che disegnata esattamente $\propto$ (ibid. 321).

70 Auf weitere Übereinstimmungen beider Texte macht Turner (ibid. 30sf.) aufmerksam.

${ }^{n}$ Ibid. 3orff. Auch Bocchi hatte sein Lob von Donatellos Georg (wie Anm, 54) nach unterschiedlichen Gesichtspunkten (costume, vivacità, bellezza) gegliedert. Diese Kategorien entsprechen zum Teil den Urteilsnormen, dic Bocchiauch in den Bellezze anwendet (vgl. Anm. 57), nicht aber jene der Kuppelbeschreibung Carlos, so daß ein Einfluß Bocchis auf Carlo unwahrscheinlich erscheint.

n Über die Verwendung des Rubrikenschema durch Mengs, Meyer und Goethe siehe: Ernst Osterkamp: Im Buchstabenbilde. Studien zum Verfahren Goethescher Bildbeschreibung, Stuttgart 1991, bes. 92-119. Ein neueres Beispiel ausführlicher Bildbeschreibung und Analyse an Hand streng getrennter Kategorien: Michael Bockemühl: Rembrandt. Zum Wandel des Bildes und seiner Anschauung im Spätwerk, Mittenwald 1981.

73 Turner (wie Anm. 67), 306. 
bekannt, der sie in seinen Vite von 1672 paraphrasiert $^{74}$. Malvasia rühmt sich 1678 der Wiederentdeckung von Agucchis sallseits begehrten< Manuskriptes, das er in der Felsina Pittrice abdruckt ${ }^{75}$. Erst in der zweiten Hälfte des 17 . Jahrhunderts scheint also die Gattung der Descrittione - ein Titelanfang, der sonst vor allem bei geographischen Schriften Verwendung findet, - auch im Falle von Kunstwerken das zum Druck nötige Interesse und Akzeptanz gefunden zu haben. Eine entscheidende und bislang kaum gewürdigte Rolle kommt dabei André Félibien $\mathrm{zu}^{76}$. Wahrscheinlich hat er während seines Italienaufenthalts $1647-$ 1649 die Texte Agucchis, Carlos oder auch weitere, vergleichbare Schriftstücke gelesen 7 . Zwischen 166I und 1677 verfaßte er dreizehn Descriptions. Ihre Bedeutung ist den Zeitgenossen nicht entgangen und die meisten von ihnen sind zu Lebzeiten

\footnotetext{
${ }^{74}$ Battisti (wie Anm. 63), 529 und Turner (wie Anm. 67), 309.
}

75 Bei der Aufzählung von Annibale Carraccis Werken schreibt Malvasia: $*$ Venere dormiente, co' scherzi d'Amore; la tanto famosa ch'egli fece in Roma al Sig. Cardinal Farnese, che meritò perciò le lodi della prima penna di quel secolo Monsig. Agucchi, nella copiosa, \& elegante sua desrizione tanto bramata al Mondo, accennata nelle Memorie Accademiche de' Signori Gelati, e che da me finalmente ritrovata, ed ottenuta, vò che si nobilmente termini, e chiuda il mio rozzo discorso $\mathrm{co}^{\text {? }}$ suo stile altrettanto sublime, e maestoso, ch'e questo* (wie Anm. 6I, sonf.). Carlos Text wurde erst im 20. Jh. gedruckt: siche Turner (wie Anm. 67), 297 .

${ }^{76}$ Uber Félibien siehe Jacques Thuiller: Pour André Félibien, Annexe: Bibliographie d'André Félibien, in: $\mathrm{Hi}-$ stoire et théorie de lart en France au XVII' siècle = XVII ${ }^{e}$ siècle, XXXV, 1983, 138, 67-95 und René Démoris: Introduction, in: Félibien: Entretiens sur les vies et sur les ouvrages des plus excellents peintres anciens et modernes, Paris $1987,8-76$, mit Besprechung der älteren Literatur. Thuillier erwähnt als einziger die Bedeutung der Beschreibung für Félibien: l'effort de Félibien sera de créer une prose qui sache décrire avec précision et commenter avec pertinence sans quitter le langage de Phonnête homme^ (80). Die erste umfangreiche Monographie über Félibien wird demnächst Stefan Germer veröffentlichen.

${ }^{77}$ Félibien forschte in den römischen Bibliotheken und sah Lanfranco bei der Arbeit zu, so Yves Delaporte: André Félibien en Italie, ses visites à Poussin et Claude Lorrain, in: Gazette des Beaux-Arts, 51, 1958, 208.

${ }_{78}^{78}$ Siehe Félibiens Bibliographie bei Thuillier (wie Anm. 76), $92 f$.

${ }^{79}$ Der Wortlaut des königlichen Dekrets vom 12, 3. 1666 ist aufschlußreich sowohl für die Einschätzung Féli- mehrfach aufgelegt worden ${ }^{78}$. Seine kunstliterarischen Verdienste brachten ihm 1666 ein Amt als »Historiographe des bastiments, peintures, sculptures, arts et manufactures royales $*$ cin, er wurde damit zum $>$ Hof-Kunsthistoriker 79 und 1673 zusätzlich Konservator der Antikensammlung des Königs $^{80}$. Die Beschreibungen Félibiens sind ferner Vorbild der seit 1667 von Colbert der Académie aufgezwungenen Vorträge geworden. Colbert ernennt Félibien zum Herausgeber dieser Conférences de l'Académie, deren berühmteste Lebruns Besprechung von Poussins Mannalese geblieben ist. Der Anteil Félibiens an diesen Beschreibungen dürfte dabei recht groß $\operatorname{sein}^{81}$.

Als Beispiel von Félibiens Beschreibungsstil ziehe ich den 1663 erstmals gedruckten Text über das monumentale Leinwandbild von Lebrun heran: Les reines de Perses aux pieds d'Alexandre, pein-

biens wie für die Erwartungen des Monarchen: * le Roy [...] voulant faire travailler à l'histoire de ses bastiments, peintures, sculptures, raretez et autres manufactures qui sont et se feront dans ses maisons royalles affin de conserver à la postérité la mémoire des grandes choses que les Roys ses prédecesseurs ont fait faire et de celles que sa Majesté fait encore faire tous les jours et dont elle enrichit ses maisons pour la gloire de son règne et l'honneur de l'estat, et ayant esté informé de la connoissance particulière que André Félibien, sieur des Avaux, s'est acquis dans l'architecture, peinture et autres arts, et de sa capacité à bien escrire sur ces matières dont il a donné des marques par plusieurs de ses ouvrages, Sa Majesté desirant favorablement traiter ledit Félibien luy a donné la charge d'historiographe des bastimens, peinture, sculptures, arts et manufactures royalles pour en jouir par luy aux gages de douze cens livres par chacun an [...] $]^{\circ}$ zit. nach Thuillier (wie Anm. 76), 72 .

So Ibid. 73 .

${ }^{8 \pi}$ André Félibien $(\mathrm{Hg}$ ): Conférences de l'Académie Royale de Peinture et de Sculpture. Pendant l'année 1667, zit. nach der Ausgabe Paris 1669 , Reprint Portland 1972. Lebruns Vortrag ist der sechste, gehalten am 5. II. 1667, 76-107. Die sorgfältig geführten Sitzungsprotokolle der Académie sind erhalten und publiziert: Anatole de Montaiglon: Procès-Verbaux de l'Académie Royale de Peinture et de Sculpture 1648-1792, Paris 1875ff. Sie tragen viel dazu bei, die Vorgänge um die Einrichtung der Conférences und ihrer Publikation durch Félibien zu verstehen. Die wichtigsten Studien hierzu sind: Bernard Teyssèdre: Roger de Piles et les débats sur le coloris au siècle de Louis XIV, Paris 1957, bes. $121-126,541-568$ u. $623-627$ und Thomas Kirchner: L'expression des passions. Ausdruck als Darstellungsproblem in der französischen Kunst und Kunsttheorie 
ture $d u$ Cabinet $d u$ Roy ${ }^{82}$. Wie Agucchi und Carlo geht auch Félibien von der Einschätzung der Beschreibung als Kopie aus: »une copie imparfaite de ce Tableau, ou plutôt une autre Peinture, qui n'a ni traits ni couleurs, mais qui servira peut-être à faire remarquer dans l'original des traits $\&$ des couleurs que l'on n'éxamine pas assez soigneusement $[\ldots] \alpha^{83}$. Funktion der Beschreibung ist es also nicht primär, das Bild zu vergegenwärtigen, von dem Gérad Edelinck 1661 ein Kupfer gestochen hatte ${ }^{84}$, sondern den Leser zu einer sorgfältigeren Betrachtung anzuleiten. Darüber hinaus ist Félibiens Text eine doppelte Lobrede: eine Würdigung des Historienbildes Lebruns und eine Huldi-

des 17. und 18. Jahrhunderts, Mainz 1991, 13-28. Das Abhalten von theoretischen Vorlesungen wird der Akademie durch Colbert seit 1667 aufgezwungen (Montaiglon, I, 315-319, 324, 339, 342f. so lautet einen Eintrag vom 30.4. 1667, bei dem ein Treffen zur ersten Conférence beschlossen wurde: ol'on ira au Cabinet du Roy pour entendre l'explication qu'en [le tableau du St. Michel de Raphael] fera Monsieur Le Brun, selon que Monsieur Colbert l'a ordonné ). Das Projet de l'ordre qui se pourroit tenir dans l'Académie $[. .$.$] , touchant les$ Conférences [...] vom 28. 3. 1667 ist den Akademieprotokollen beigelegt. Es trägt zustimmende Randnotizen in Colberts Handschrift sowie seine Unterschrift. Das Dokument könnte von Félibien in Absprache mit Colbert verfaßt worden sein. Dort heißt es: $»$ Dans le besoin de trouver une plume digne de cette occupation, il semble que la Compagnie auroit à souhaiter que Mr. Félibien, qui asseurément, outre le stile qu'il a très excellent, possède encore toutes les qualitès nécessaires à cet employ, ayant donné des marques au public des connoissances qu'il a de la Peinture et de la Sculpture, fust en état de pouvoir s'engager dans ce travail « (Montaiglon, I, 316). Félibien wird dann auch am 30, 4, 1667 von Colbert in die Akademie eingeführt und nimmt an ihren Sitzungen als $*$ Conseiller honnorèr * teil (ibid. 318 , siche auch 324). Nach der Publikation des ersten Bandes dieser Vorträge beklagen sich die Mitglieder der Akademie über Fehler und drängen Colbert, ihnen eine größere Kontrolle über die von Félibien zu publizierenden Texte einzuräumen. Daraus kann entnommen werden, daß Félibien bei der Redaktion der Vorträge viel Eigenes einfließen ließ. Möglicherweise aufgrund dieses Dissens unterblieb die Publikation weiterer Vorträge. (Sitzungen des 26. 5. 1668, ibid. 33rf. und 20. 4 . 1669 , ibid. 339).

${ }^{82}$ Paris 1663 , wiederabgedruckt in André Félibien des Avaux: Descriptions de divers ouvrages de peinture faits pour le Roy, Paris 1671 und in Ders.: Recueil de descriptions de peinture et d'autres ouvrages faits pour le Roy, Paris 1689, Reprint Genève 1973. Nach dieser gung des Sonnenkönigs, dem die Schrift gewidmet ist. Félibiens Verknüpfung von Werk und Auftraggeber geht über den ikonologischen Bezug von altem und neuem Alexander hinaus ${ }^{85}$. Einerseits verdanke sich das Gelingen dieses einmaligen Werkes den Anregungen Ludwigs XIV. ${ }^{86}$ Andererseits beweise der König durch seine daraufhin von allen geteilte Zustimmung für eben dieses Bild, daß sein >gottgegebener Geschmack dem der übrigen Menschheit überlegen sei ${ }^{87}$.

Wie Carlo zuvor, gliedert Félibien seine Beschreibung nach verschiedenen Rubiken, deren Reihenfolge er auch reflektiert ${ }^{88}$. Der Schriftsteller fängt mit der Erklärung der Ikonographie an (sujet, 3of.).

Auflage wird hier zitiert. Zu dem Bild Lebruns siche: Charles Le Brun 1619-1690, Katalog einer Ausstellung, Versailles 1963, 70-73, Nr. 27 (mit Abbildung).

${ }^{8}$ Félibien: Recueil (wie Anm. 82), 29.

${ }^{84}$ A. P. F. Robert-Dumesnil: Le Peintre-Graveur français, VII, Paris $1844,200 f f ., ~ N r .42$. Es handelt sich um eine große, seitenverkehrte Reproduktion (zwei Platten, zusammen $678 \times 900 \mathrm{~mm}$.). Abbildung bei Teyssèdre (wie Anm. 81), Abb. 5. Über die politische Ikonologie des Bildes und des Textes Félibiens siehe auch René Démoris: Le corps royal et l'imaginaire au XVH' siècle: le Portrait du Roy par Félibien, in: Revue des sciences humaines, 172 , 1978.4 und Ders: Peinture et histoire: Félibien et la stratégie du récit historique au siècle de Louis XIV (d'après les Reines de Perse aux pieds d'Alexandre), in: Jean Bissière (Hg.): Récit ct histoire, Paris 1984, 23-35.

${ }^{85}$ Lebruns Bild ist anscheinend die erste Alexanderallegorese Ludwigs: Ekkehard Mai: Poussin, Félibien und Le Brun. Zur Formierung der französischen Historienmalerei an der Académie Royale de Peinture et de Sculpture in Paris, in: Ders. u. a. (Hg.), Historienmalerei in Europa. Paradigmen in Form, Funktion und Ideologie, Mainz 1990, 21-24.

86 -ce rare Ourrage que son excellent Auteur vient d'achever, est moins une production de son art \& de sa science, qu'un effet des belles idées qu'il a receûé de V[otre] M[ajesté] quand elle luy a commandé de travailler pour Elle. ₹ Félibien: Recueil (wie Anm. 82), $28 f$.

${ }^{87}$. [copie imparfaite de ce tableau... qui servira...] a a faire connoistre que Dieu qui met dans l'ame des Rois \& des Princes des Vertus extraordinaires, leur donne encore une intelligence si parfaite de toutes choses, qu'ils en jugent mieux que tous les autres hommes, puis que l'approbation de V[otre] M[ajesté] est suivie de celle de tous les sçavans, \& mesme de tout le monde. $\ll$ ibid. 29.

${ }^{88}$ So beispielsweise: $*$ Comme la disposition d'un'Tableau est la partie que l'on doit considérer la première; je diray [...] $\times$ ibid. 3 i. 
Er unternimmt dann ein »récit général « des Bildes, indem er jede einzelne Figur und ihre Kleidung schildert (disposition, 31-34). Dann vertieft sich Félibien in die Beschreibung des Ausdrucks und der Handlung dieser Figuren (expression des passions, 35-52), der Verteilung und Behandlung des Lichtes (lumière, 52-54) und der Farben (couleurs, $54-62)^{89}$. Weitere kunsttheoretische Kategorien erwähnt er im abschließenden Lob (62-66), ohne sie jedoch beschreibend zu präzisieren: dessein (62), proportion (64), convenance (65). Félibien erweitert also Carlos Rubriken. Er benützt dabei Kategorien, die die Kunsttheorie des Cinquecento und vor allem Franciscus Junius (De pictura veterum, 1637) als die Teile der Malerei besprechen. In der bereits 1662 publizierten I dée de la Perfection de la Peinture hat Roland Fréart die Anwendung ähnlicher Kategorien vorgeführt. Inhalt seiner Schrift sind die fünf $\rightsquigarrow$ Parties fondamentales de la Peinture $\alpha$ : invention, proportion, couleur und lumière, expression des passions, perspective. Sie sollen die Beurteilung eines jeden Gemäldes ermöglichen, und Fréart zeigt ihre Anwendung am Beispiel mehrerer Stiche, die er ausführlich bespricht. Ihm kommt es jedoch nicht auf die Beschreibung, sondern auf den Beweis der Richtigkeit der von ihm aufgestellten Regeln an und auf die sich daraus ergebende Beurteilung. Dementsprechend klein bleibt der beschreibende Teil des Textes Fréarts. Diese Kategorien gewinnen einen paradigmatischen Stellenwert für die Kunsttheorie, indem sie die Grundlage der Doktrin der Pariser Académie Royale de Peinture

${ }^{89}$ Die Behauptung, daß Félibiens Beschreibungen sich *hauptsächlich mit Bildgegenständen * befassen würden (Manfred Boos: Französische Kunstliteratur zur Malerei und Bildhauerei 1648 und 1669 . DDas Gesuch des Martin de Chamois« (1648) und André Félibiens - Vorwort \& zu seiner Conférences-Ausgabe (I669), München (Dissertation) 1966,72 ) ist eine Verkennung einer ihrer historisch wichtigsten Aspekte.

${ }^{\circ}$ Der Versuch der dogmatischen Festlegung tritt schon sehr früh in der Geschichte der Akademie ein. So heißt es im Zusammenhang eines ersten Versuches zur Einrichtung von Conférences am 30. August 1653: - le temps n'a peu permettre de former entièrement la table des matières concernant la peinture, après en avoire desterminé les quatre partie principalle, quy sont le traict, le joure et ombre, la coulleure et et de Sculpture werden ${ }^{90}$. Félibien scheint sich jedoch nicht an dieser Dogmatisierung beteiligt zu haben. Er weiß, daß die Kunst sich nicht in einem strengen Regelkodex subsumieren läßt: »quelquefois il faut se conduire contre les regles ordinaires de l'art $\alpha$, »pour [...] donner [aux parties d'un tableau] plus de force, plus de beauté et plus de grâce ${ }^{91}$. Ihn interessiert die Analyse des Einzelwerkes genauso wie die Destillierung abstrakter Kategorien. In der Einleitung der Conférences fordert er im Namen Colberts eine Verbindung von Regeln und Exempla: $:$ Il [Colbert] dit que dans les Sciences et les Arts il y a deux manières d'enseigner, sçavoir, par les preceptes \& par les exemples, que l'une instruit l'entendement, \& l'autre l'imagination [...] pour bien instruire la jeunesse dans l'Art de peindre, il seroit donc necessaire de leur exposer les ouvrages des plus sçavans Peintres, $\&$ dans des Conferences publiques, faire connoistre ce qui contribue le plus à la beauté \& à la perfection des Tableaux. Que chacun ayant la liberté de dire son sentiment l'on feroit un examen de tout ce qui entre dans la composition d'un sujet, \& mesme que les avis differens qui se pourroient rencontrer, serviroient à découvrir beaucoup de choses qui seroient autant de preceptes $\&$ de maximes ${ }^{92}$. Während der Akademie-Direktor Charles Le Brun und dessen Sekretär Henri Testelin sich um die Festlegung eines abstrakten Regelwerkes der Kunst bemühen, bleibt die Betonung des Exempels und damit der Bedeutung der Beschreibung das Verdienst Félibiens.

l'expression, l'on a remis d'en régler les particularitéz aux assemblée suivante. « Zitiert nach: Montaiglon (wic Anm. 81), I, 76. Die endgültige Festlegung dieser Doktrin erfolgt in den tables de préceptes, die der Sekretär der Akademie, Henri Testelin, seit 1675 vorträgt und I680 publiziert. Siehe André Fontaine: Les doctrines d'art en France. Peintres, amateurs, critiques. De Poussin à Diderot, Paris 1909; Teyssèdre (wie Anm. 81), ${ }_{63} \mathrm{I}$ und Kirchner (wie Anm. 81), 10-28, mit älterer Literatur.

${ }^{9}$ Félibien: Entretiens sur les vies et sur les ouvrages des plus excellents peintres anciens et modernes, zit. nach Kirchner (wie Anm. 81), 17. Dort auch eine Darstellung der Differenzen zwischen Félibien und der Akademie.

${ }^{92}$ Félibien (wie Anm. 81), Préface, unpaginiert [4f.]. 
Die Beschreibung von Bildern nach katalogisierten Rubriken, wurde in der zweiten Hälfte des 17. Jahrhunderts nicht nur in Paris üblich. Bellori bespricht in seiner in den $1680 \mathrm{oer}$ Jahren entstandenen Beschreibung von Marattas Apoll und Daphne: argomento (257), disposizione universale (257-264), invenzione (zum Verhältnis von Bild und Poesie $264-268)$ sowie, disegno e colore $(268-271)^{93}$. Noch 1766 beschreibt Giovanni Battista Casanova, der damalige Direktor der Dresdner Akademie und Bruder des sprichwörtlichen Abenteurers, Mengs' Hochaltarbild für die Dresdner Hofkirche nach einem ähnlichen Schema: Erfindung und Idee (134f.), Zusammensetzung (composizione), Helldunkles, Wirkung (136ff.), Wabl, Charakter $u$. Ausdruck (138-142), Gewänder (142f.), Zeichnung (143), Farbengebung (143f.) $)^{94}$.

1770 äußert Salomon Gessner in seinem Brief über die Landschaftsmalerei zwei Wünsche, deren Erfüllung für die ^Aufnahme der Kunst - gemeint ist vor allem die Ausbildung von Künstlern - er größte Bedeutung beimißtos. Zuerst soll ein Werk verfaßt werden, in dem »die Grundregeln der Kunst kurz, und so deutlich als möglich, vorgetragen und erklärt, und dann auf besondere Fälle angewandt seyn ${ }^{96}$. Der zweite Wunsch ist, »daß ein Werk entstehen möchte, worin, in jeder Art Mahlerkunst, die besten Werke umständlich beschrieben, und nach allen Regeln des Schönen untersucht und beurtheilt würden; allein es müssten Werke seyn, die in Kupfer gestochen sind $\star^{97}$. Gessner bevorzugt aus praktischen Über-

${ }^{93}$ Gianpietro Bellori: Dafne trasformata in lauro. Pittura del Signor Carlo Maratti. Dedicata a' Trionfi di Luigi XIV. il Magno. Descritta in una lettera ad un Cavaliere Forastiero, in: Ottavio Lioni: Ritratti di alcuni celebri pittori del secolo XVII..., Roma 173r. Über Belloris Beschreibungen vgl. Oskar Bätschmann: Giovan Pietro Belloris Bildbeschreibungen, in: Boehm u. Pfotenhauer (wie Anm. 13, 279-3n). Nach Bätschmann ist die Beschreibung von Marattas Bild bereits 1681 (297, Anm. 64) oder 1684 (280) entstanden.

${ }_{94}$ Giovanni Battista Casanova: o. T. (vermischte Nachrichten), in: Neue Bibliothek der schönen Wissenschaften und der freyen Künsten, III, 1, 1766, 132-144. Vgl. August Langen: Kunstwerke in dichterischer Deutung. Eine Auswahl von Winckelmann bis Burckhardt, Dresden $1940,240$.

9s Salomon Gessner: Brief über die Landschaftsmahlerey legungen heraus jene Werke, deren Reproduktionsstiche snicht rar, und, so viel möglich, nicht kostbar sind. So würd' es immer ein leichtes seyn, solche in den Sammlungen an jedem Ort zu finden, oder sie selbst anzuschaffen $\alpha^{98}$. Die zu beschreibenden Bilder müssen andererseits "nur die besten Werke aus jedem Alter [...] nur solche, worinn die Regeln des wahren Schönen mit dem besten Verstand angebracht sind, und aus denen sie vorzüglich deutlich gemacht werden können ${ }^{99}$. Gessner greift hier die Argumente Félibiens wieder auf ${ }^{\circ 00}$. Noch deutlicher als Félibien erwartet er, daß Beschreibung nicht Abbild, sondern Erläuterung eines Werkes sein soll. Casanovas Beschreibung erwähnt er als vorbildlich ${ }^{101}$.

In der Zeit, in der Gessner seinen Brief verfaßte, wurde die Möglichkeit, Bilder sprachlich wiederzugeben, zunchmend in Frage gestellt. Der $*$ Diskurs der Repräsentation « gerät in eine Krise. Lessing, der sich theoretisch in seiner Abhandlung Über die Grenze der Malerei und Poesie dieser Frage nähert, schreibt 1766 , daß sich das, was die Maler durch Linien und Farben am besten ausdrücken können, durch Worte gerade am schlechtesten ausdrücken läßt $\star^{\text {TO2 }}$. Lessing spricht überhaupt der Rede-sofern sie poetisch sein will - »das Vermögen ab, ein körperliches Ganzes nach seinen Teilen zu schildern*. Beschreibungen dürfen nur stattfinden, »wo es daher auf das Täuschende nicht ankömmt, wo man nur mit dem Verstande seiner Leser zu tun hat, und nur auf deutliche und soviel möglich vollständige Begriffe gehet $\alpha^{103}$.

an Herrn Fuesslin, den Verfasser der Geschichte der besten Künstler in der Schweiz, zitiert nach: Sämtliche Schriften, hg. v. Martin Bircher, III, Zürich $1974,267$.

${ }_{96}^{6}$ Ibid. 268.

97 Ibid. 27 If.

$9^{8}$ Ibid. 269.

99 Ibid. 272.

${ }^{100}$ Die Belehrung von Künstlern durch Regeln und Exempel ist ein Gedanke, der bereits in der Florentiner Accademia del Disegno im ausgehenden 16. Jahrhundert formuliert wurde: Boos (wie Anm. 89), 58 .

${ }^{100}$ Gessner (wie Anm. 95), 273.

${ }^{102}$ Gotthold Ephraim Lessing: Laokoon oder über die Grenzen der Malerei und Poesie, Berlin 1766 , zit. nach Stuttgart 1987, Kap. XX, ist.

${ }^{109}$ Ibid., Kap. XVII, 126. 
Diderot wird durch die Praxis der Kunstkritik auf das selbe Problem gestoßen. Im Salon von 1767 bemerkt er, daß, je detaillierter eine Beschreibung ist, desto mehr Verwirrung sie in der Einbildungskraft des Lesers verursacht ${ }^{104}$. Anläßlich eines Ruinenbildes Hubert Roberts heißt es beispielsweise: $»$ Voilà une description fort simple, une composition qui ne l'est pas moins et dont il est toutefois très difficile de se faire une juste idée, sans l'avoir vue $[\ldots]$ vingt artistes feraient vingt tableaux où l'on trouverait les objets que i'ai indiqués, et à peu près aux places que je leur ai marquées, sans se ressembler entre eux ni à l'esquisse de Robert. Qu'on l'essaye? et que l'on convienne de la nécessité d'un croquis. Le plus informe dira mieux et plus vite, du moins sur l'ordonnance générale, que la description la plus rigoureuse et la plus soignée $\alpha^{105}$. Diderots Bedenken sind grundsätzlicher Art. Von 1769 an verzichtet er auf die ausführlichen Beschreibungen, die den Reiz seiner früheren und noch heute berühmteren Salons ausgemacht hatten ${ }^{\text {Io6 }}$.

Möglicherweise ist Winckelmann bereits vor Lessing zu ähnlichen Einsichten gelangt: Helmut Pfotenhauer hat darauf hingewiesen, daß er im Laufe seines Lebens zwei gegensätzliche Formen von Beschreibungen anwendet. Einerseits die *Beschreibungen nach dem Ideal«, wie er seine berühmten literarischen Nachschöpfungen bezeichnet, bei denen beispielsweise aus dem verstümmelten Torso vom Belvedere die lebendige

${ }^{104}$ Diderot (wic Anm. 26), XVI, 285 u. 343. Vgl. Albert Dresdner: Die Entstehung der Kunstkritik im Zusammenhang der Geschichte des europäischen Kunstlebens (1915), zit. nach der Neuauflage München 1968, 216.

Ios Diderot (wie Anm. 26), XVI, 248. Den Nachweis der Unmöglichkeit einer getreuen - also rückübertragbaren - Ubersetzung von Bildern in Sprache hat, sofern dies noch überhaupt nötig war, Max Dessoir erbracht, indem er Seminarteilnehmer nach Beschreibungen Zeichnungen erstellen ließ: Anschauung und Beschreibung, in: Archiv für systematische Philosophie, X, 1904, 20-65.

${ }^{106}$ Vgl. Langen (wie Anm. 25), $329 f$. Bukdahl (wie Anm. 25), 30r führt die Reduktion der Beschreibungen auf einen entsprechenden Wunsch seitens Friedrich Melchior Baron von Grimm, Herausgeber der Correspondance littéraire, zurück. Die von ihr angeführten Belegstellen überzeugen jedoch nicht, da Diderot in den Salons Grimm häufig als rein fiktiven Gesprächspart-
Gestalt eines Herakles und die Erinnerung an seine Taten hervorleuchten ${ }^{107}$. Diese Texte werden zunehmend von den Beschreibungen "nach der Kunst $\propto$ abgelöst: Beschreibungen, deren Aufmerksamkeit den bildhauerischen Eigenschaften der Werke gilt, wie auch deren stilitischen Merkmalen, die durch Vergleich mit anderen Statuen festgestellt werden ${ }^{108}$. Wilhelm Heinses eigentümliche Mischung einer sinnlich-erotischen und zugleich übergenauen Beschreibung der Düsseldorfer Rubens-Gemälde (1777) können dagegen als Versuch gewertet werden, die von Lessing aufgestellte Grenze zu überwinden ${ }^{109}$.

\section{Die wissenschaftliche Beschreibung}

Diese Reflexion und Infragestellung der Möglichkeit der Beschreibung geht zeitlich einer zunehmenden Veränderung der technischen und soziologischen Bedingungen voraus, unter denen Kunstbeschreibungen verfaßt werden. Die Conférences der Pariser Akademie der zweiten Hälfte des 17. Jahrhunderts sind im wesentlichen für werdende Künstler von lehrenden Künstlern vorgetragen. Für Gessner waren die Adressaten der von ihm erwünschten Beschreibungen gleichermaßen werdende $\mathrm{Maler}^{\mathrm{nO}}$, denen sich allerdings auch "Liebhaber « hinzugesellen" ${ }^{\mathrm{m}}$. Als Verfasser kamen nur wenige, "grösseste Kenner« in Frage. Unter den Lebenden benennt er namentlich neben Casanova zwei weitere Akademieprofessoren: Dietrich

ner einführt, um die drohende Monotonie des Monologs durch Dialog aufzulockern.

${ }^{307}$ Johann Joachim Winckelmann: Kleine Schriften, Vorreden, Entwürfen, hg. v. Walther Rehm, Berlin 1968, 169-173. Der Beschreibung folgt der Satz: $\times$ Nach dieser Idealischen Beschreibung würde die nach der Kunst folgen * (173).

${ }^{108}$ Pfotenhauer (wie Anm. 18), bes. $326 \mathrm{ff}$.

${ }^{109}$ Johann Jakob Wilhelm Heinse: Über einige Gemählde der Düsseldorfer Gallerie. An Hern Canonicus Gleim, in: Der Deutsche Merkur, 1777, Mai 17 -135 und Juli 6o9o. Vgl. Walther Killy: Die Sprache der Bildbeschreibung, in: Zeitschrift für Kunstgeschichte, 44, 1981, $6 \mathrm{ff}$; Gotifried Boehm: Anteil. Wilhelm Heinses *Bildbeschreibung*, in: Helmut Pfotenhauer (Hg), Kunstliteratur als Italienerfahrung, Tübingen 1991, 21-39; Pfotenhauer (wie Anm, 18), 32 off.

${ }^{170}$ Gessner (wic Anm. 95), 267 .

III Ibid. 272. 
und Oeser, sowie den mit ihm befreundeten Künstlerdilettanten, Sammler und Generaldirektor der Künste in Dresden, Christian Ludwig von Hagedorn $^{\text {II }}$. Diderots berühmte Salonkritiken ( 1759 bis $178 \mathrm{I}$ ) sind in Friedrich Melchior Grimms Correspondance littéraire erschienen, einer handschriftlich (!) in wenigen Exemplaren vervielfältigten Zeitschrift. Erst im Verlauf des 19. Jahrhunderts nimmt das an den bildenden Künsten interessierte Publikum stark zu, zugleich wird der kritische Umgang mit Kunstwerken Gegenstand eines neuen Berufes. Der öffentliche Charakter der Kunstsammlungen wächst, Museen und Ausstellungen ziehen wachsende Zahlen von Besuchern an. Die Erfindung und Anwendung neuer Verfahren verwandelt die Reproduktionsgraphik in ein Massenmedium. Umrißstich, Holzstich und Lithographie, später die Photographie, gepaart mit verschiedenen photomechanischen Druckverfahren verbreiten die Kenntnis der immer berühmter werdenden Werke" ${ }^{\text {II }}$. Mit dem wachsenden Interesse für aktuelle und historische Kunst verbindet sich die Entstehung und Institutionalisierung des Faches Kunstgeschichte. Diese Entwicklung hat sich in Europa mit unterschiedlichen Schwerpunkten vollzogen ${ }^{\mathrm{I} 4}$. Charakteristisch für den deutschsprachigen Raum war dabei die allmähliche Ausbildung einer universitären Disziplin, die von beiläufigen kunsthistorischen Vorlesungen (in Kiel seit $\left.{ }^{1770}\right)^{\mathrm{n} 5}$ über die Ernennung von Ordinarien für Kunstgeschichte (erstmals in Göttingen 1813) bis hin zur Einrichtung kunstgeschichtlicher Seminare und Institute (in Bonn seit 1872) führte ${ }^{116}$. Die - heute international anerkann-

I12 Ibid. 273 .

${ }^{113}$ Ettore Spalletti: La documentazione figurativa della opera d'arte, la critica e l'editoria nell'epoca moderna (1750-1930), in: Giovanni Previtali ( $\mathrm{Hg}$ ): Storia dell'arte italiana, I, 2, Torino $1979,415-484$.

${ }^{14} 4$ Einen Uberblick gibt Georg Kauffmann: Die Entstehung der Kunstgeschichte im 19. Jahrhundert, Opladen 1993.

us Hans Tintelnot: Kunstgeschichte, in: Geschichte der Christian-Albrechts-Universität Kiel 1665-1965, 5.1, Neumünster 1969 , 17 of.

${ }^{n 6}$ Heinrich Dilly: Kunstgeschichte als Institution. Studien zur Geschichte einer Disziplin, Frankfurt a. M. 1979; Wolfgang Beyrodt: Kunstgeschichte als Universi- te - Institutionalisierung der Kunstgeschichte als akademisches Fach bedingt eine Verlagerung des Sprechens und Schreibens über Kunst aus Künstlerateliers, Liebhaberkabinetten und literarischen Zirkeln in einen vom Anspruch der ,Wissenschaftlichkeit beherrschten universitären Rahmen. Eine Verlagerung, von der die historischvergangene Kunst am meisten betroffen ist.

Eine Folge dieser Spezialisierung ist die Vertiefung des von Moritz aufgezeichneten Grabens zwischen Dichtung und Geschichte. Aus der Sicht des Dichters schadet die Kunstbeschreibung eines Winckelmanns der Betrachtung, weil sie den Blick vom Ganzen abgezogen, und auf das Einzelne geheftet hat ${ }^{\mathrm{H}} \mathrm{7}$. An ihre Stelle setzen deswegen Moritz, Wackenroder und Schlegels Waller das Bildgedicht. Dies ist nur einer der Wege des poetischen Umgangs mit Bildern nach der Krise der ${ }^{-R e p r a ̈ s e n t a t i o n ~}{ }^{n 18}$. Auf weitere Formen literarischer Bildbeschreibung im 19. und 20. Jahrhunderts kann hier nicht eingegangen werden. Zwar gibt es weiterhin viele Dichter, die zu einzelnen Werken der bildenden Kunst Stellung nehmen. Ihre Aussagen verlieren sich aber in der Masse. Es sind einige wenige literarische Texte inmitten einer Flut neu entstehender Sach- und Fachliteratur $^{119}$. Seit der Mitte des 19. Jahrhunderts nimmt die Anzahl von Kunsthistoriker-Bildbeschreibungen explosionsartig zu. Ein Beispiel: Michelangelos Skulpturen der Medici-Kapelle wurden seit dem I6. Jahrhundert bis 1850 weniger als zehnmal, zwischen 1850 und 1930 dagegen von über vierzig verschiedenen Autoren, meist Wissenschaftlern, einer genauen Beschreibung unterzo-

tãtsfach, in: Peter Ganz u. a. (Hg.): Kunst und Kunsttheorie 1400-1900, Wiesbaden 1991, 313-333; Sybille Dürr: Zur Geschichte des Faches Kunstgeschichte an der Universität München, München 1993 beinhaltet ein Verzeichnis der Lehrstuhlinhaber aller kunsthistorischen Institute des deutschsprachigen Raumes seit ihrer Gründung ( $145-154)$. Leider weist diese Ubersicht vicle Lücken und Fehler auf.

${ }^{17} 7$ Moritz (wic Anm. 14), 95f.

${ }^{118}$ Die neuere Tradition des Bildgedichts skizziert Pestalozzi (wie Anm. 14), 569-591. Siehe auch das Handbuch zu dieser Gattung: Gisbert Kranz: Das Bildgedicht, 3 Bände, Köln und Wien 1981-1987. 
gen; die Häufigkeit nimmt nach 1930 wieder etwas ab. Anscheinend schwächt sich nach dem ersten Drittel unseres Jahrhunderts die Bedeutung $a b$, die das Fach Kunstgeschichte der Beschreibung beimißt. Kehren wir aber zu den Anfängen der wissenschaftlichen Bildbeschreibung zurück. Sie fußen auf der skizzierten Tradition Agucchis, Carlos, Félibiens und der Connaisseur-Beschreibungen des 18 . Jahrhunderts, unterscheiden sich jedoch in wichtigen Punkten:

I. Bis zur Mitte des 19. Jahrhunderts werden Beschreibungen häufig von Aussagen über die eigentliche Unfähigkeit der Sprache, dem Werk der bildenden Kunst gerecht zu werden, begleitet. Dies gilt insbesondere, seitdem im 17 . Jahrhundert der Anspruch einer genauen Repräsentation aufgestellt wurde ${ }^{120}$. Der Kunstwissenschaftler dagegen, dessen Beruf die Auslegung der nun als erklärungsbedürftig angesehenen Werke ist, setzt der sprachlichen Erkenntnis kaum Grenzen. Er beansprucht für seine - mittels der Beschreibung getroffenen Aussagen - Wahrheitsstatus. Ab jetzt sind wissenschaftliche Dispute über die Ausle-

${ }^{19}$ Ein Uberblick deutscher Texte, der noch wesentliche Teile des 19. Jahrhunderts erfaßt, gibt Langen (wie Anm. 94). Verschiedene Aspekte diskutieren die Aufsätze vorì Günther Hess, Hans Körner, Victor I. Stoichita, Gerhard Neumann, Elisabeth Schmid und Ernst Osterkamp in: Boehm u. Pfotenhauer (wie Anm. 13).

${ }^{\mathrm{T} 20}$ Hier nur wenige Beispiele: Agucchi (wie Anm. 6I) $\rightarrow[. .$. ne [Carraccis Venusbild] distesi perciò questa piacevole descrittione: con la difficoltà, che le opere singolari à imaginare, non che isprimer si possono da un debole ingegno. Onde, conoscendo io di non essermi avvicinato, ne per molto spatio al vero; assai crederò haver fatto, se nella maniera, che le pitture da un großo velo coperte-malagevolmente si discernono da riguardanti: cosi poteßero le presenti essere egualmente da i lettori apprendute $\left(\mathrm{S}_{0}\right)$ ); whi a minuto descriverne le [die einzelne Teile des Gesichtes] volesse, non si prenderebbe per avventura una lunga, mà una forte difficile impresa; perchè troppo più perfette elle sono che la penna à dichiarare non si arriva (soff.); Félibien (wie Anm. 82), 35 v'ay peine à trouver des termes assez propres pour en [Lebruns Bild] bien décrire la beauté; \& je crains de gaster par la foiblesse de mes paroles, ces nobles expressions que le Peintre a si dignement représentées par la force de son génie.«; Raffaelo Del Bruno: Ristretto delle cose più notabili della città di Firenze (1689), zitiert nach Firenze ${ }^{2} 1698,53 *$ E di vero chi può lodare a bastanza l'eccellenza, la maestà, la grazia, e la gung eines Werkes möglich und üblich, während Beschreibungen, wie sie Schlegel in Gesprächsform formuliert hatte, undenkbar werden, da der Kunsthistoriker im Gegensatz zum Dichter seinen Gesichtspunkt absolut setzt. Der Wissenschaftler ist um Genauigkeit, nicht selten um Vollständigkeit bemüht. Dies artet gelegentlich in Akribie aus, in Texte, die alles aussprechen wollen ${ }^{121}$.

2. Lob und Tadel eines Werkes sind - als unhistorische und unwissenschaftliche Kategorien - nicht länger Anlaß einer Beschreibung. Mit dem Historismus werden absolut gesetzte Normen des Kunsturteils aufgegeben. Die aus historischer Perspektive ausgesprochene ,Würdigung` mag dann das frühere Lob ersetzen. Obwohl Sympathie und Antipathie der Wissenschaftler nicht immer verdeckt werden können, bemühen sich die Beschreibungen um ,Charakterisierung statt Beurteilung. 3. Im $*$ Zeitalter der technischen Reproduzierbarkeit« entfällt für die Sprache die Funktion des Bildersatzes. Mit zunehmender Häufigkeit befindet sich neben dem beschreibenden Text eine Ab-

vaghezza di questa fabbrica [Michelangelos MediciKapelle]? Tentarono già molti eruditi Scrittori di descrivere distintamente le sue bellezze, ma non giungendo ad una minima parte, diedero a dividere, che nelle lodi di Michelangelo, e di quest'opera insigne, era manchevole, ed insufficente, fino l'istessa Eloquenza. «; Ernst Moritz Arndt: Reisen durch einen Theil Deutschlands, Italien und Frankreichs in den Jahren 1798 und 1799 , Leipzig 180I, $321 *$ Doch das Vortreffliche liegt hinter der Sprache«; Die Beteuerung des Unsagbarkeitstopos ist seit dem letzten Drittel des 19 . Jahrhunderts viel seltener.

${ }^{122}$ Beschreibungen in der Länge der oben besprochenen Texte Agucchis oder Félibiens waren im 17. Jahrhundert Ausnahmen und sind es bis heute geblieben. Sieht man von solchen Einzelfällen ab, ist eine Tendenz zu immer genaueren Beschreibungen festzustellen. Stellt man die Beschreibungen eines bestimmten Werkes zusammen, zeigt sich, daß die ausführlichsten häufig im Zeitraum von 1870 bis 1930 entstanden. Bei Tizians Assunta übertrifft beispielsweise der Text von Giovanni Battista Cayalcaselle und Joseph Archer Crowe: Tiziano, la sua vita e i suoi tempi con alcune notizie della sua famiglia, I, Firenze $1877,178-186$ alle älteren Beschreibungen. Theodor Hetzer: Die frühen Gemälde des Tizian. Eine stilistische Untersuchung, Basel 1920, 24-28 geht noch etwas weiter, verweilt bei jedem der Aposteln und gibt eine detailliertere Kompositionsanalyse als alle Vorgänger. 
bildung des Werkes ${ }^{122}$. Nicht die Beschreibung, sondern die Abbildung vermittelt die Anschauung des fernen Originals. Der Text wendet sich nicht mehr an die Einbildungskraft, sondern an das Auge des Lesers. Beschreibung hat immer mehr jene Rolle zu erfüllen, auf die Félibien und Gessner bereits hingewiesen hatten: sie soll eine Anleitung zur Betrachtung, eine Sehhilfe zum Verständnis des Werkes sein.

4. Neben dem Dargestellten gewinnt mit der Professionalisierung der Autoren die Beschreibung der Darstellungsweise an Gewicht. Die entsprechenden Kategorien der barocken Beschreibungen (Komposition, Zeichnung, Farbe) erhalten zunehmende Bedeutung, während der für Félibien so zentrale Aspekt der »expression des passions « vernachlässigt wird. Die in der Goethezeit beliebte szenische Beschreibung von Gemälden nimmt im Laufe des Jahrhunderts stark $a b^{123}$.

Abgesehen von diesen allgemeinen Tendenzen haben Kunsthistoriker ihren Beschreibungen sehr unterschiedliche Formen und Schwerpunkte gegeben. Trotz wissenschaftlicher Objektivität zeichnet der persönliche Beschreibungsstil den Kunsthistoriker aus. Der Kunsthistoriker eignet sich durch Beschreibung seinen Gegenstand an, und es überrascht nicht, daß er diesem Teil seiner Arbeit häufig große Bedeutung beimißt. Nicht selten kann bei Vertrtern unseres Faches - im Laufe ihres wissenschaftlichen Werkes - eine Veränderung des Beschreibungsstiles festgestellt werden.

${ }^{122}$ Vgl. Michael Baxandall: Patterns of intention, New Haven und London 1985, 8f. Evelina Borea: Giovan Pietro Bellori e la scomodità delle stampec, in: Elisabeth Cropper u. a. (Hg.): Documentary Culture Florence and Rome. From Grand-Duke Ferdinand I to Pope Alexander VII, Bologna 1992, 269 sieht den Argomento. della Galleria Farnese dipinta da Annibale Carraci disegnata e intagliata da Carlo Cesio, nel quale spiegansi e riduconsi allegoricamente alla moralita le favole poetiche in essa rappresentate (Roma 1657 ) als sil primo caso di libro dedicato a un capolavoro dell'arte costituito di testo storico-artistico e di stampe relative all'oggetto. Bellori erläutert die Verteilung der Bilder, die Bedeutung des Programmes und den Inhalt der einzelnen Bilder, ohne diese jedoch zu beschreiben. Die Zusammenstellung von Text und Bild ist erst nach 1800 üblich: Kauffmann (wie Anm. 114), 37 f.
Als Beispiel dafür bietet sich Jacob Burckhardt an, in dessen Schriften sich der Übergang zur wissenschaftlichen Bildbeschreibung erst relativ spät niederschlägt. Ich beschränke mich auf einen Vergleich zwischen dem Cicerone (185s in erster Auflage) und zwei seiner späteren kunsthistorischen Schriften, nämlich Das Altarbild und die Erinnerungen aus Rubens aus den Neunziger Jahren ${ }^{124}$.

Burckhardts Umgang mit Beschreibung ist sehr reflektiert. Er nimmt in der Einleitung des Cicerone ausführlich dazu Stellung: »das Beschreiben war nur insoweit meine Aufgabe, als es dazu dienen konnte, auf wesentliches Detail aufmerksam zu machen, oder die Auffindung und Erkennung der betreffenden Gegenstände zu erleichtern; sonst rechnete ich durchgängig darauf, daß der Leser das in Rede stehende gesehen habe oder sehen werde ${ }^{125}$. Burckhardt scheint also der Beschreibung keine allzu große Bedeutung beizumessen. Auf der darauffolgenden Seite fügt er die häufig zitierten Sätze hinzu: Das Räsonnement des , Ciceroner macht keinen Anspruch darauf, den tiefsten Gedanken, die Idee eines Kunstwerkes zu verfolgen und auszusprechen. Könnte man diesen überhaupt in Worten vollständig geben, so wäre die Kunst überflüssig, und das betreffende Werk hätte ungebaut, ungemeißelt, ungemalt bleiben dürfen. Aber auch bis an dic erlaubten Grenzen bin ich nicht gegangen; schon die notwendige Kürze verbot dies. Das Ziel, das mir vorschwebte, war vielmehr: Umrisse vorzuzeichnen, welche das

${ }^{123}$ Timm (wic Anm. 28), 351.

${ }^{124}$ Die ausführlichste Untersuchung von Burckhardts Beschreibungsstils hat Stefan Kummer geleistet: Kunstbeschreibungen Jacob Burckhardts im Cicerone und in der Baukunst der Renaissance in Italien, in: Boehm und Pfotenhauer (wie Anm. 13), 357-372. Kummer beschränkt sich allerdings auf Burckhardts frühere Werke. Der hier vorgenommene Vergleich möge als Verwirklichung von Kummers Schlußsatz gelten: „Daß sich Burckhardts ästhetische Überzeugungen gegen das Lebensende wandelten, dürfte nicht streitig sein - man denke nur an die zunehmende Hochachtung vor dem Barock. Inwiefern dieser Wandel sich in seinen späteren Kunstbeschreibungen niederschlug, werden künftige Forschungen zeigen. $\alpha$

${ }^{125} \mathrm{Zit}$. nach Jacob Burckhardt: Gesamtausgabe, hg. v. Heinrich Wölfflin u. a., Stuttgart u. a. 1929-34, III, I. 
Gefühl des Beschauers mit lebendiger Empfindung ausfüllen könnte ${ }^{126}$.

Auffällig ist tatsächlich die Kürze aller Burckhardtschen Beschreibungen. Er schließt sich der Tradition des Unsagbarkeitstopos an und versucht nicht einmal Wallers `Geist` darzustellen. Er distanziert sich von vornherein von der Strategie vieler seiner Zeitgenossen, die eine vollständige Erfassung von Kunstwerken durch Ausführlichkeit anstreben. In der treffsicheren Kürze liegt gerade Burckhardts Vorzug ${ }^{127}$. Ihr ist sicherlich auch zu verdanken, daß seine Schriften noch heute als unerschöpfliche Fundgrube von Zitaten benützt werden.

Als Beispiel für die Analyse seines Beschreibungsstiles bietet sich Tizians Assunta an. Burckhardt hielt sie für das Hauptwerk des Venetianers und hat zweimal seine Gedanken über das Altarbild der Frari-Kirche ausformuliert ${ }^{128}$. Der erste Text, im Cicerone, lautet: »Die untere Gruppe ist der wahrste Glutausbruch der Begeisterung; wie mächtig zieht es die Apostel, der Jungfrau nachzuschweben! In einigen Köpfen verklärt sich der tizianische Charakter zu himmlischer Schönheit. Oben, in dem jubelnden Reigen, ist von den erwachsenen Engeln der, welcher die Krone bringt, in ganzer, herrlicher Gestalt gebildet; von den übrigen sieht man nur die überirdisch schönen Köp$\mathrm{fe}$, während die Putten in ganzer Figur, ebenfalls in ihrer Art erhaben, dargestellt sind. Wenn Correggio eingewirkt haben sollte, so ist er doch hier an wahrer Himmelsfähigkeit der Gestalten weit übertroffen. Der Gottvater ist von weniger idealem Typus als die Christusköpfe Tizians; vom Gürtel an verschwindet er in der Glorie, welche die Jungfrau umstrahlt. Sie steht leicht und sicher auf den noch ideal, nicht mathematisch wirklich gedachten Wolken; ihre Füße sind ganz sichtbar. Ihr rotes Gewand hebt sich ab von dem gewaltig wehenden, vorn geschürz-

${ }^{126}$ Ibid. 2 .

${ }^{12} 7 \mathrm{Vgl}$. Kummer (wie Anm. 124), 362f.

${ }^{22} 8$ Werner Kaegi: Jacob Burckhardt. Eine Biographie, VI, Basel u. a. 1977, 626f. diskutiert Burckhardts Einschätzung des Bildes und zitiert Auszüge ciner dritten $\mathrm{Be}$ schreibung aus dem Vorlesungsmanuskript Notizien zur ten dunkelblauen Mantel, ihr Haupt ist umwallt von ganz mächtigen Haaren. Der Ausdruck aber ist eine der höchsten Divinationen, um welche sich die Kunst glücklich zu preisen hat: die letzten irdischen Bande springen; sie atmet Seligkeit $\alpha^{\text {r29 }}$.

Vom gleichen Gemälde heißt es in der posthum verlegten Schrift Das Altarbild: $*[\ldots]$ ähnlich verhält es sich mit der herrlichsten Assunta, welche je geschaffen worden, mit derjenigen des Tizians [...] Über der Jungfrau erscheint [...] Gott Vater, welchem ein rasch herbeischwebender Engel voll Andacht eine Krone bringt [...] Schon die Verteilung des Herganges innerhalb des (ziemlich hohen) Formates ist die denkbar vollkommenste: über einem einheitlichen Sockel (der Apostelgruppe) folgt ein mächtiges, mit heller Glut erfülltes Rund (unten umzogen durch die Putten und Engel des Wolkenkranzes, oben durch Cherubsköpfe); und vor dieser Glut die Assunta, und in die Glut verschwimmend Gott Vater ${ }^{130}$.

Wie nach der Einleitung zum Cicerone zu erwarten war, versucht keiner dieser Texte dic Anschauung des Bildes zu ersetzen. Beide sind Anleitungen für den Leser. Doch hier fängt auch ihr offensichtlicher Unterschied an. Die Beschreibung des Cicerone ist einerseits eine Anleitung zur Beurteilung der einzelnen Teile des Bildes ${ }^{131}$. Die Gestaltung der Körper und vor allem jene der Köpfe wird an dem Sujet, an der »Himmelsfähigkeit «, gemessen und fast durchweg für sehr gut befunden. Tizian wird vermerkt - überbietet sogar ein mögliches Vorbild, Correggio. Solche Vergleiche unterschiedlicher Maler mit dem Zweck der Beurteilung haben eine lange Geschichte in der Sprache des Connaisseurs. So schreibt Casanova von dem Dresdner Hochaltarbild: »In der schönen Figur der Magdalena hat Mengs die ganze Grazie des Correggio angebracht, aber der Charakter ist ed-

italienischen Kunst seit dem XV. Jabrhundert (Blatt 267).

${ }^{129}$ Burckhardt (wie Anm. 125), IV, 341.

${ }^{230}$ Burckhardt (wie Anm. 125), XII, Iorf.

${ }^{13}$ Kummer (wie Anm, 124), 363 $\times$ Es wird evident, daß seine [Burckhardts] Beschreibungen das Werturteil zum primären Ziel haben $\alpha$. 
ler, und die Zeichnung reiner ${ }^{{ }^{132}}$. Andererseits vermittelt die Beschreibung des Cicerone die Begeisterung des Autors für eines seiner Lieblingsbilder und den Eindruck, den die verschiedenen Teile auf ihn machen; Burckhardt scheint vom Glutausbruch der Apostel getroffen, mit ihnen der Jungfrau nachschweben zu wollen. Diese Beschreibungsform erklärt den Untertitel des Cicerone: Eine Anleitung zum Genuß der Kunstwerke Italiens. Dabei ist Genuß als die Erfahrung der Teilhabe durch sinnliche Wahrnehmung zu verstehen $^{133}$. Im Schlegelschen Gespräch sagt beispielsweise Louise: Die Mahlerey macht es einem leichter, sie zu genießen, sie spricht so unmittelbar in unsre Sinnenwelt hinein. $\alpha$ und auf die Frage Reinholds: $\cdots \mathrm{Ja}$, was nennen Sie so etwa genießen? $\propto$ antwortet sie: »Mich der schönen Darstellung erfreuen, mich daran sättigen, sie ganz in mich aufnehmen ${ }^{134}$.

Die Sprache der späteren Beschreibung ist dagegen nüchterner, obgleich Burckhardt das Bild mit einem Superlativ einführt. Die Apostelgruppe ist nicht mehr »der wahrste Glutausbruch der Begeisterung* sondern schlicht ein seinheitlicher Sokkel. Überhaupt hat sich die Perspektive der Beschreibung von den Figuren und der Handlung hin zur Komposition verlagert, vom Gegenstand zur Form und andererseits, von dem Hinweis auf »wesentliches Detail « hin zur Erfassung des Bildganzen. Beschreibungen, die stets vom Bildganzen ausgehen und der geometrisch-bildflächenbezogenen Komposition eines Bildes besondere Achtung schenken, sind nicht nur im Altarbild, sondern auch in den Erinnerungen aus Rubens die Regel.Von Rubens' Laurentiusmarter (München,

${ }^{132}$ Casanova (wic Anm. 94), 141. Vergleiche mit Stilen anderer Maler hatte auch Dolce der bereits zitierten Beschreibung der Assunta angeschlossen.

${ }^{13} 3$ Zum Begriff des Genusses bei Burckhardt siehe Wilhelm Schlink: Jacob Burckhardt über den *Genuß der Kunstwerke», in: Trierer Beiträge, II, Juli 1982, 47-55; Gottfried Boehm: Anteil. Wilhelm Heinses $*$ Bildbeschreibung $\propto$, in: Helmut Pfotenhauer $(\mathrm{Hg}$.): Kunstliteratur als Italienerfahrung, Tübingen 1991, bes. $26 \mathrm{f}$. und Klaus Herding: ^... Woran meine ganze Seele Wonne gesogen... $\propto$ Das Galericerlebnis - eine verlorene Dimension der Kunstgeschichte?, in: Peter Ganz u. a.
Alte Pinakothek), die mit jener Tizians (aus der Jesuitenkirche in Venedig) verglichen wird, heißt es beispielsweise: Der große Venetianer hatte sich auf die Schönheit der Einzelnen, zumal des auf dem Rost ausgestreckten Körpers, auf seine drei verschiedenen Lichter, auf einen großen baulichen Hintergrund u. a. Reizmittel verlassen können; Rubens dagegen machte den Heiligen, welcher auf dem Rost sitzend durch Schergen zurückgebogen wird, zur genauen Mitte einer stark bewegten und durch eine große Horizontale doch optisch beruhigten Komposition, welche gegenüber der lockeren Anordnung Tizians die feste Geschlossenheit für sich hat; alle Ursachen sind mächtiger und die Wirkung einheitlich ${ }^{135}$. In den Abschnitten über die Komposition des flämischen Malers ist die Beschreibung der Bildgeometrie noch ausführlicher ${ }^{136}$.

Die Unmittelbarkeit des Eindrucks, wie ihn die Beschreibungen des Cicerone spiegeln, geht also in diesen späteren Beschreibungen verloren. Während der Cicerone zum Teil den Erwartungen Louises entsprechen würde, könnten die $\mathrm{Be}$ schreibungen der späten kunstgeschichtlichen Schriften der Feder Reinholds entsprungen sein. Statt von einer »Anleitung zum Genuß k könnte man in den letzteren von einer $\gg$ Anleitung zur Betrachtung « sprechen. Diese Bezeichnung verwendet Burckhardt 1874 in seiner programmatischen Antrittsvorlesung zu dem neu gegründeten Basler Lehrstuhl für Kunstgeschichte ${ }^{137}$. Mit der Veränderung in Burckhardts Verhältnis zur bildenden Kunst, auf die bereits hingewiesen worden ist ${ }^{138}$, geht folgerichtig auch ein Wandel seiner Beschreibungssprache einher. Spezifische Ursachen

(Hg.): Kunst und Kunsttheorie $1400-1900$, Wiesbaden 1991, $257-85$, bes. 277 f.

${ }^{134}$ Athenäum (wic Anm. I), 45.

${ }^{155}$ Burckhardt (wic Anm. 125), XIII, 449.

${ }^{136}$ Eine Charakterisierung solcher Beschreibungen Burckhardts am Beispiel von Rubens' Raub der Leukippiden, geben Killy (wie Anm. 109), ff. und Pfotenhauer (wie Anm. 18), 1995, $329 f$.

137 Uber die Kunstgeschichte als Gegenstand eines akademischen Lehrstuhls, Vortrag gehalten am 6. Mai 1874 , in: Burckhardt (wie Anm. 125), XIII, 23.

${ }^{138} \mathrm{Kaegi}$ (wie Anm. 128), VI, 539 u. a. deutet dies bereits an, 
dieses Wandels sind vielfältig. Ein entscheidender Einfluß dürfte die jahrelange, kunstgeschichtliche Unterrichtspraxis des Universitätsprofessors gespielt haben: die Form der Beschreibung des Cicerone eignet sich zwar zur Mitteilung von persönlichen Eindrücken, nicht aber zur akademischen Lehre, von deren Inhalten Wissenschaftlichkeit und damit Objektivierung erwartet werden. Mit der Lehre geht auch der zunehmende Einsatz von Abbildungen, insbesondere von Photographien einher, die Burckhardt vor allem in der zweiten Hälfte der I870er Jahren leidenschaftlich erwirbt und im Verlauf seiner Studienreisen kistenweise nach Basel verschickt. Photographien sind $\mathrm{ihm}$ sowohl ein unentbehrliches Lehrmittel für seine kunsthistorischen Vorlesungen als auch in wachsendem Maß Grundlage seiner "Studien ${ }^{139}$. Die auf über zehntausend Stück angewachsene Photosammlung trägt ihren Teil an dem auffällig distanzierteren Verhältnis zu den Werken: auf einer verkleinerten Schwarzweiß-Reproduktion fällt die Komposition stärker ins Auge als vor dem originalen Werk und - darauf hat schon Malraux hingewiesen ${ }^{140}$ - die Gemeinsamkeiten zwischen den im Format angeglichenen, nebeneinander gleichwertig aufgereihten Werken sind zuungunsten ihrer Eigenart gesteigert. Für den >Altersstilk Burckhardts sind auch die veränderten Arbeitsbedingungen verantwortlich. Die späten kunsthistorischen Schriften sind am Basler Schreibtisch des aufgrund seines Gesundheitszustandes nicht mehr reisefähigen Gelehrten entstanden. Der Cicerone dagegen, der $>$ zu zwei Dritteilen während der Reise geschrieben wur-

beschreibt aber vor allem den Geschmackswandel Burckhardts; Dieter Jaehnig: Kunsterkenntnis bei Jacob Burckhardt, in: Deutsche Vierteljahresschrift für Literaturwissenschaft und Geistesgeschichte, 58,1984 , 23f.; Herding (wie Anm. 133), 277f. und ausführlich Martina Sitt: Kriterien der Kunstkritik. Jacob Burckhardts unveröffentlichte Ästhetik als Schlüssel seines Rangsystems, Wien u. a. 1992, 17fff. mit Diskussion weiterer Literatur.

${ }^{239}$ Kaegi (wic Anm. 128), VI, 300-305.

$4^{\circ}$ André Malraux: Psychologie de l'Art, r. Le Musée Imaginaire, Genève 1947, 24 .

${ }^{24}$ Burckhardt (wic Anm. 125), III, 2.

${ }^{142}$ Timm (wie Anm. 28), 345. Die vergleichende Beschrei- de $\kappa^{I 4 I}$, spiegelt die Betrachtung der Originale wider.

Solche Faktoren wirken sich nicht nur auf Burckhardt aus. Der hier skizzierte Wandel in der Form der Bildbeschreibung ist typisch für viele Zeitgenossen des Basler Professors. Bezeichnend für das Ende des 19 . Jahrhunderts ist die zunchmende Geometrisierung des Beschreibungsvokabulars sowie die Verbreitung von vergleichenden Beschreibungen, wie es Burckhardt mit der Gegenüberstellung Tizian-Rubens praktiziert ${ }^{1 / 2}$. Während die Erwähnung Correggios im zitierten Auszug des Cicerone lediglich dazu dient, den Rang Tizians hervorzuheben, werden in den späteren Schriften vergleichbare Werke einander gegenübergestellt, um durch ihre Unterschiede, das Besondere, den je eigenen Charakter deutlicher $\mathrm{zu}$ machen, was allerdings ein Werturteil nicht ausschließt. Vieles in diesen späten Beschreibungen Burckhardts erinnert an die seines Nachfolgers Heinrich Wölfflin. In einer Hinsicht erweist sich jedoch der Basler Altmeister als rückständig. Zwar spielt bei den späteren Beschreibungen die Bewertung eine etwas geringere Rolle und der Bewertungsgegenstand hat sich dort zum Teil verändert. Bewertet werden häufig nicht mehr nach klassizistischer Manier Figuren und Köpfe, sondern das Bildganze. Grundsätzlich hält aber Burckhardt an einer am Jahrhundertende unter Kunsthistorikern zunehmend verpönten normativen Ästhetik fest.

Die am Beispiel Burckhardts skizzierte Entwicklung zu einer wissenschaftlichen Beschreibung ist auch ein Verlust: Verlust der unmittelbaren Kunsterfahrung ${ }^{\text {I3 }}$. Viele Kunsthistoriker haben

bung wurde allerdings nicht erst durch Balthasar Speth cingeführt, sic kommt beispielsweise bereits in Winckelmanns Geschichte der Kunst des Altertums vor: Johann Joachim Winckelmann: Sämtliche Werke, Donaueschingen 1825, Neudruck Osnabrück $1965, \mathrm{Bd}$. IV, 139f. $(V, 1, \S 40)$.

${ }^{143}$ Die Kritik dieser Sprache ist fast so alt wie die Sprache selbst: $*$ Reinhold: Kennen Sie Mengs Beschreibung dieser [Correggios] Madonna? Louise: O ja! Sie enthält alles, was den Mahler angeht, und was ich übergehen mußte, weil ich es nicht verstehe, und weil grade dabey Worte ohne den Anblick nicht helfen * Athenäum (wie Anm. 1), 94. Je crois sincèrement que la meilleure critique est celle qui est amusante et poétique; non pas 
diese Schattenseite ihres Ausdrucksmediums empfunden und sich um eine Sprache bemüht, die auch diesen Aspekten des Werkes gerecht wird. Ohne den Versuch unternehmen zu wollen, die vielseitige Geschichte der Kunstbeschreibung im 20. Jahrhundert zu behandeln, seien hierfür drei Beispiele zitiert.

In seiner Dissertation von ris benennt Theodor Hetzer Bewegung und Pathos von Tizians Assun$t a$, die der Cicerone eindrücklich beschworen hatte. Allerdings relativiert sie Hetzer durch Bezugnahme auf den historischen Hintergrund: $» D$ as Bild ist unerhört im damaligen Venedig. Es hat einen Reichtum an Bewegung wie keines vor ihm und wie kaum ein zweites von Tizian. Das Pathos der Judith und der [Paduaner] Fresken ist ins Imposante gesteigert $\alpha^{144}$.

Im Verlauf unseres Jahrhunderts pendelt die Beschreibung der Wirkung von Bildern zwischen dem evokativen, expressionistischen Schreibstil eines Edmund Hildebrandt und der um Objektivierbarkeit bemühten, analytischen Fachsprache eines Max Imdahl:

Edmund Hildebrandt über Michelangelos Tag: »Ein gigantischer Männerleib im Vollbesitz unermeßlicher Kräfte liegt der Giorno hingewälzt auf seinem engen Lager wie eine drohende Lawine. Mit gewaltigem Ruck emporfahrend enthüllt der

celle-ci, froide et algébrique, qui, sous prétexte de tout expliquer, n'a ni haine ni amour, et se dépouille volontairement de toute espèce de tempérament $\alpha$ Charles Baudelaire: Salon de 1846 , zitiert nach Hans Körner: Der imaginäre Fremde als Bildbetrachter, in: Boehm u. Pfotenhauer (wie Anm. 13), 402.
Riese die Muskelberge seines Rückens und seiner Schultern, während einer der mächtigen Arme in die Tiefe greift und zur Hälfte unseren Blicken entschwindet: ein Motiv, das im Verein mit dem wie drohend hinter dem Rücken aufragenden, unvollendeten, Haupte mit wahrhaft niederschmetternder Gewalt auf den Beschauer einstürmt * ${ }^{145}$. Max Imdahl über Giottos Gefangennabme Christi: Man muß, exemplarisch, hinweisen auf eine Schräge, die von einer Keule zur Linken durch die Köpfe von Jesus und Judas hindurch auf den Zeigegestus des Pharisäers zur Rechten hinführt. [...] Zugleich ist die Schräge die Bedingung einer semantischen Komplexität, wenn man beachtet, daß Jesus zum einen in durchaus passiver und unterlegener Rolle von Judas umfangen wird, $[\ldots]$ daß aber zum anderen Jesus den Judas an Körpergröße überragt, daß er in durchaus aktiver und überlegener Rolle auf Judas herabblickt Auge in Auge und daß das Blickgefälle von Jesus auf Judas herab aufgenommen und zu einem bildbeherrschenden Ausdruck erhoben ist durch eben jene Schräge. Die Schräge ist eine der wichtigsten szenischen Sinn ergebenden Erfindungen in Giottos Bild der Gefangennahme, denn in ihr sind offensichtliche Daten der Unterlegenheit und der Überlegenheit Jesu wechselseitig ineinander transformiert ${ }^{146}$.

144 Hetzer (wie Anm. 121), 25 .

${ }^{145}$ Edmund Hildebrandt: Michelangelo. Eine Einführung in das Verständnis seiner Werke, Leipzig und Berlin 1913,84 f.

${ }_{146}{ }^{6}$ Max Imdahl: Giotto. Arenafresken. Ikonographie, Ikonologie, Ikonik (1980), München ${ }^{2} 1988,93$. 